\title{
Stereoselectivity of the hydrogenation of galactofuranosyl exoglycals
}

\author{
Audrey Caravano, ${ }^{a}$ Weidong Pan, ${ }^{c}$ and Stephane P. Vincent**a, \\ ${ }^{a}$ Ecole Normale Superieure, Departement de Chimie, CNRS UMR 8642, 24 rue Lhomond, \\ 75231 Paris Cedex 05, France \\ ${ }^{c}$ University of Namur, Laboratoire de Chimie-Bioorganique, Rue de Bruxelles 61 B-5000 \\ Namur, Belgium \\ Email: Stephane.vincent@fundp.ac.be \\ Dedicated to Professor Alain Krief
}

\begin{abstract}
This work describes a study of the $\alpha / \beta$-diastereoselectivity of the hydrogenation of phosphonylated exoglycals in the galactofuranose series. Nine exoglycals displaying sterically hindering groups on the $\alpha$ or the $\beta$ face have been synthesized and hydrogenated using Pearlman's catalyst. These reactions gave the expected $C$-glycoside with good to excellent $\alpha$ selectivities.
\end{abstract}

Keywords: Exoglycal, C-glycoside, hydrogenation, phosphonate

\section{Introduction}

In the course of our study involving the exploration of the mechanism of glycosyl processing enzymes, we have developed an approach to generate $C$-glycosidic phosphonates in the galactofuranose series (Galf). First we developed conformational probes mimicking UDP-Galf the substrate of key enzymes implied in the biosynthesis of the mycobacterial cell wall: UDPgalactose mutase (UGM) and Galactofuranosyl transferases. ${ }^{1,2}$ We then prepared nucleotideexoglycals, fluorinated ${ }^{3}$ or not, ${ }^{4}$ that displayed interesting time-dependent inactivation properties against UDP-galactose mutase.

In our studies, exoglycals became key intermediates: for instance we found that once protected with four TBDMS groups, the enol ether could be hydrogenated selectively to give a $\alpha$ phosphonate (Scheme 1) thus yielding a $C$-glycosidic analog of the natural Galf-1- $\alpha$-phosphate. ${ }^{4}$ In some cases, glycoconjugates whose anomeric configuration is inverted compared to the natural substrate displayed interesting inhibition properties. ${ }^{5}$ A survey of the literature in the Galf series showed that there is no published method to synthesize the corresponding Galf-1- $\beta$ - 
phosphonate. Therefore, we addressed the question of the obtention of $\beta$-phosphonates from phosphonylated exoglycals.

The purpose of this study is to define the $\alpha / \beta$ stereoselectivity of the hydrogenation as a function of the protective group pattern of the phosphonylated exoglycal. Since both $\alpha$ and $\beta$ diastereomers can be transformed into biologically relevant molecules, we found it interesting to assess the scope of these hydrogenations as a function of $i)$ the selective steric hindrance of one of the two faces of the exoglycal ii) the directing effect of a $\mathrm{OH}$ group at the 2- or the 3- or the 5position of the galactofuranosyl moiety (scheme 1). In the early eighties, it had already been acknowledged that hydroxyl-directed hydrogenations are of considerable generality. ${ }^{6,7}$ The addition of hydrogen usually occurs from the same face of a cyclic system than the directing group. ${ }^{8,9}$ Thus, we developed the chemistry to differentiate the 2 - and the 3-positions of the starting exoglycal and selectively install a hindering group, or a $\mathrm{OH}$ group, on either face of the carbohydrate (Scheme 1).

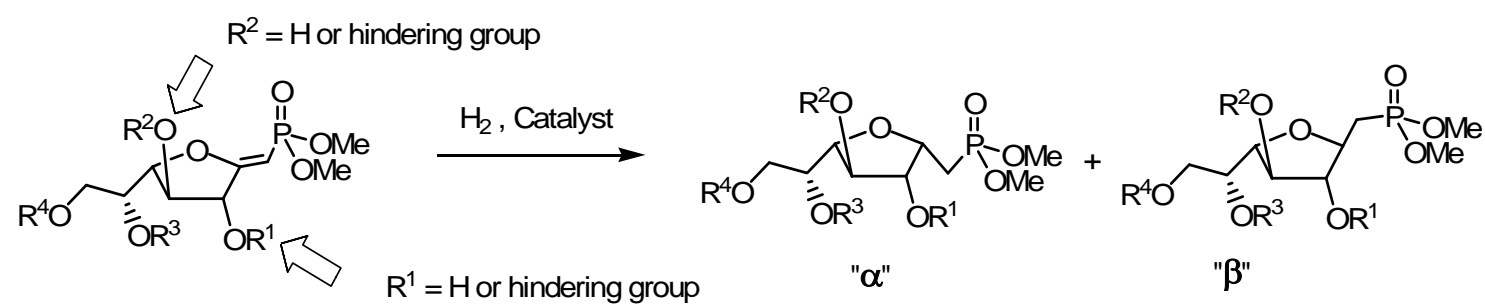

Scheme 1

\section{Results and Discussion}

\section{Selective protections of the intermediate lactones}

First, we developed a synthetic pathway for selectively protecting the 3-position of the starting d-1,4-galactonolactone.

The synthesis starts from known 5,6-O-(dimethyl-methylidene)-d-galactono-1,4-lactone 1 (Scheme 2). ${ }^{10}$ Despite the steric hindrance of the 5 and 6 positions, alcohol 1 was selectively silylated in presence of a slight excess of TBDMSCl giving 2 in $85 \%$ yield. This regioselectivity may be explained by the fact that the carbonyl group of the lactone decreases the $\mathrm{pKa}$ of the adjacent secondary alcohol, thus increasing its reactivity towards electrophiles. Intermediate alcohol 2 was transformed into lactone 3 (in 93\% yield) by addition of pivaloyl chloride. This key intermediate is of particular interest since all the secondary alcohols in lactone $\mathbf{3}$ are differentiated. Under published conditions $\left(\mathrm{AcOH} / \mathrm{H}_{2} \mathrm{O}, 60^{\circ} \mathrm{C}\right),{ }^{10}$ the acetonide could be hydrolyzed to afford an intermediate 5,6-diol 4 in an expected $60 \%$ yield. This moderate yield can be explained by the concomitant deprotection of the TBDMS group at the 2-position (the resulting triol 5 was isolated in 8\%). However, we had planned to install two TBDMS groups at 
positions 5 and 6: therefore, instead of isolating intermediate diol 4, we performed the persilylation on the crude hydrolysis mixture yielding 6 in $74 \%$ yield for two steps.
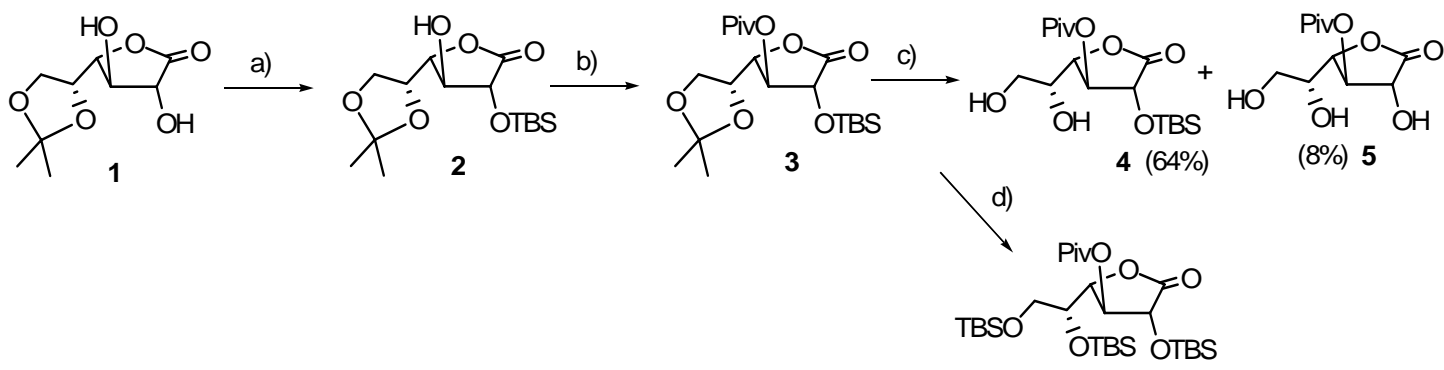

6

Scheme 2. a) TBDMSCl, Im, DMF (85\%) b) PivCl, $\mathrm{Py}, \mathrm{CH}_{3} \mathrm{CN}, 80^{\circ} \mathrm{C}(93 \%)$ c) $\mathrm{AcOH}, \mathrm{H}_{2} \mathrm{O}$, $60^{\circ} \mathrm{C}$. d) conditions $\mathrm{c}$ then TBDMSCl, Im, DMF, $60^{\circ} \mathrm{C}(74 \%)$.

\section{Synthesis of the phosphonylated exoglycals}

From lactones $\mathbf{3}$ and $\mathbf{6}$ we could synthesize two exoglycals $\mathbf{7}$ and $\mathbf{8}$ according to the one-pot procedure developed by Lin et al. in 72 and $76 \%$ yield, respectively (Scheme 3). ${ }^{11,12}$
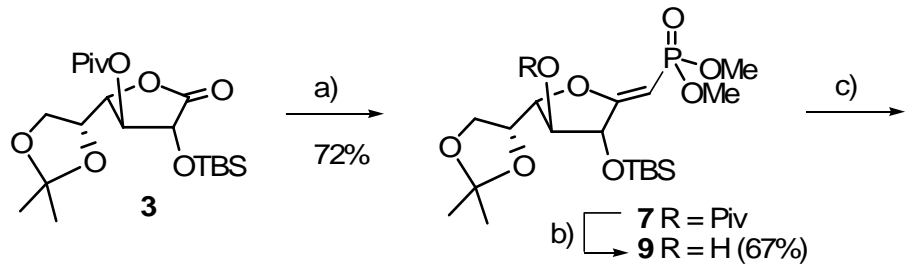

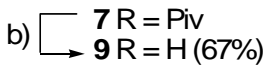
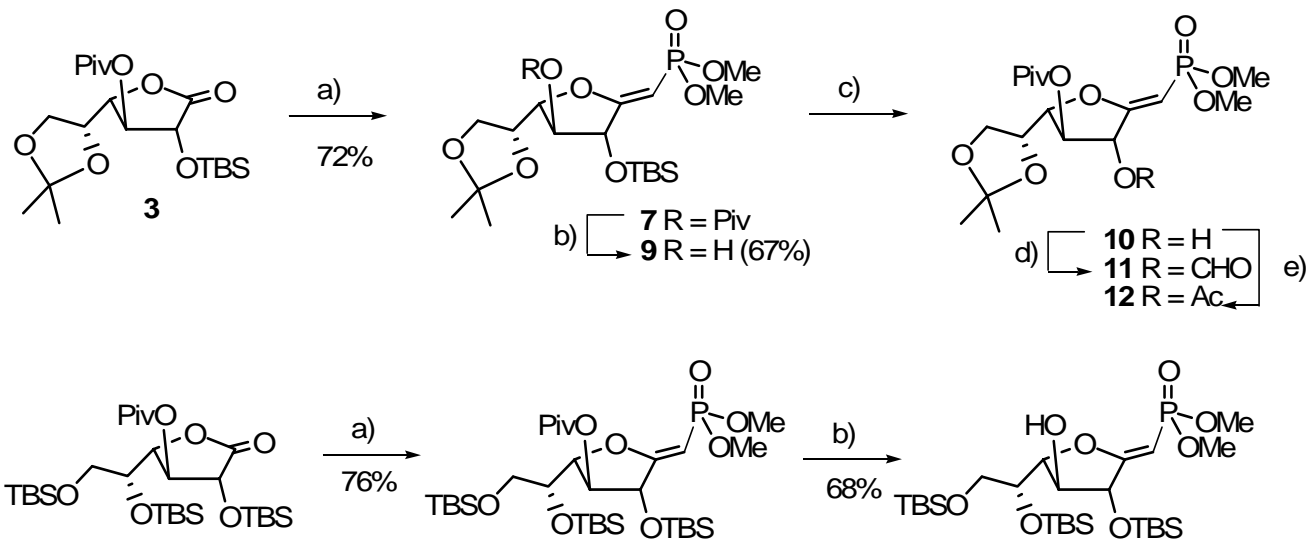

6

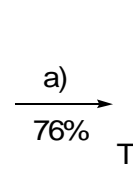

TBSO
8

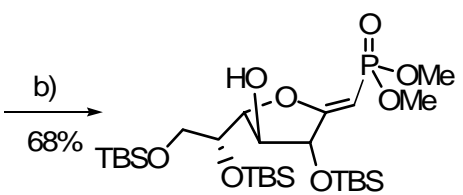

13

Scheme 3. a) $\mathrm{LiCH}_{2} \mathrm{PO}(\mathrm{OMe})_{2}$ then $\left(\mathrm{CF}_{3} \mathrm{CO}\right)_{2} \mathrm{O}$, Py. b) $\mathrm{MeOH}, \mathrm{NaH}, \mathrm{RT}$. c) TBAF, THF (73\%). d) $\mathrm{DCC}, \mathrm{HCO}_{2} \mathrm{H}, \mathrm{RT}(79 \%)$. e) $\mathrm{Ac}_{2} \mathrm{O}, \mathrm{Py}, \mathrm{RT}(96 \%)$.

Since their protective groups patterns were orthogonal, the two key molecules $\mathbf{7}$ and $\mathbf{8}$ could easily be transformed into a broad range of exoglycals presenting different functional groups on all the four available alcohols of the galactofuranose moiety. The pivaloates could be chemoselectively hydrolyzed without deprotection of the TBDMS, the acetonide or the phosphonate methyl esters, to give alcohol 9 and 13 in 67 and 68\% yield, respectively. A TBAF deprotection could in turn lead to alcohol $\mathbf{1 0}$ that was further acylated into formate $\mathbf{1 1}$ and acetate 12. At this stage we had in hand seven exoglycals (molecules 7 to 13) that could be used in 
hydrogenation assays. This series of molecules can be divided into two families: the first one displays a sterically hindering TBDMS group at the 2-position ( $\alpha$ face of the exoglycal) and the second one presents a pivaloate at the 3 -position hindering the $\beta$-face. Moreover, we also tested exoglycals 14 and 15 (Figure 1) whose synthesis had been previously described. ${ }^{1,4}$

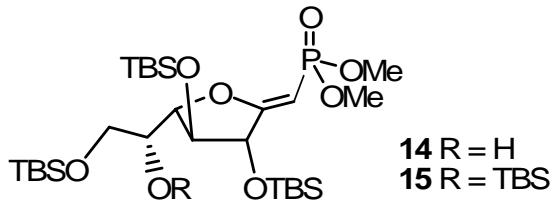

\section{Figure 1}

\section{Hydrogenations of the exoglycals}

The results of the hydrogenation experiments from exoglycals $\mathbf{7}$ to $\mathbf{1 5}$ are summarized in Table 1. The structures of the major products and some side-products are represented in Figure 2.

\section{Major Products}

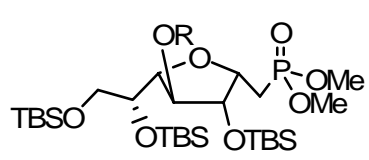

8a: $R=$ Piv

$13 \mathbf{a}: R=H$

15a: $R=$ TBS

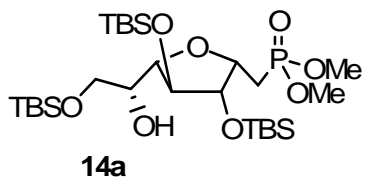

\section{Side-products}

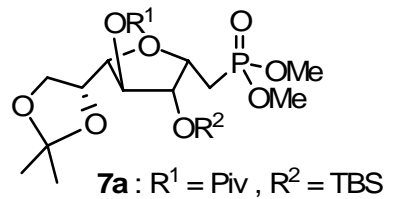

9a: $R^{1}=H, R^{2}=T B S$

10a: $\mathrm{R}^{1}=$ Piv, $\mathrm{R}^{2}=\mathrm{H}$

11a: $R^{1}=$ Piv,$R^{2}=C H O$

12a: $R^{1}=$ Piv,$R^{2}=A c$

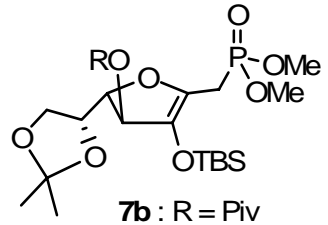

9b: $\mathrm{R}=\mathrm{H}$
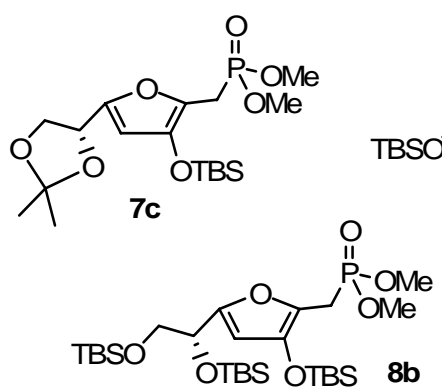

\section{Figure 2}

We first had to find the most efficient catalyst for this reaction (Table 1, entries 1 to 4 ). Exoglycal 13, presenting a totally hindered $\boldsymbol{\alpha}$ face and no bulky group on the $\boldsymbol{\beta}$ face was selected for this series. Interestingly, under high hydrogen pressure (100 bars) and vigorous stirring, the saturation of the enol ether was only observed with Pearlman's catalyst (entry 4), yielding ether 13a in $89 \%$ yield. A single diastereomer was observed by ${ }^{1} \mathrm{H}$ and ${ }^{31} \mathrm{P}-\mathrm{NMR}$ of the crude final reaction mixture. The expected $\alpha$ configuration was unambiguously determined by the $\mathrm{H} 1-\mathrm{H} 2$ coupling constant $\left(J_{1-2}=4.8 \mathrm{~Hz}\right)$, indicating a cis relationship between these two protons. For $\beta$ configured galactofuranosides, the $J_{1-2}$ value would have been lower than $1.0 \mathrm{~Hz} .{ }^{13,14}$ Moreover, 
a NOESY experiment clearly showed a correlation between protons $\mathrm{H}-1$ and $\mathrm{H}-4$ of $\mathbf{1 3 a}$, thus confirming the $\alpha$-configuration. Other standard homogeneous or heterogeneous catalysts (entries 1-3) either did not produce any product or yielded $10 \%$ of unidentified side-products. We then systematically performed this reaction with $\mathrm{Pd}(\mathrm{OH})_{2}(0.4$ equivalent/w) in AcOEt at room temperature under $\mathrm{H}_{2}$ atmosphere (1.5 bar). Good to excellent yields (72 to 95\%) have been obtained for the 9 starting exoglycals (entries 5 to 13). It should be noted that some side-products could be formed (Table 1, entries 5, 7,8 and 11). We could isolate and characterize some of them (structures depicted in Figure 2). Interestingly, these molecules resulted from a migration of the double bond to give 2-alkoxy-endoglycals. In one case, we could even isolate a furan 7c, likely resulting from an elimination of dihydrofuran $\mathbf{7 b}$. These side-reactions are quite unusual and consequently the yields were not quantitative. Nevertheless, replacing AcOEt by $\mathrm{MeOH}$ as the solvent improved the yield and decreased the amount of side-products (entry 6).

Table 1. Hydrogenations of exoglycals 8-15

\begin{tabular}{|c|c|c|c|c|c|c|c|c|c|}
\hline Entry & Substrate & Catalyst & Solvent & $\begin{array}{c}\mathrm{H}_{2} \\
\text { (bar) }\end{array}$ & Yield $^{\mathrm{a}}$ & $\alpha / \beta^{b}$ & Product & $\begin{array}{c}J_{1-2} \\
(\mathrm{~Hz})^{\mathrm{c}}\end{array}$ & $\begin{array}{c}\text { Side } \\
\text { products }\end{array}$ \\
\hline 1 & 13 & $\mathrm{Pd} / \mathrm{C}$ & AcOEt & 100 & 0 & - & $-{ }^{d}$ & - & $-{ }^{d}$ \\
\hline 2 & 13 & $\mathrm{PtO}_{2}$ & AcOEt & 100 & 0 & - & - & - & $-\mathrm{e}$ \\
\hline 3 & 13 & $\left(\mathrm{Ph}_{3} \mathrm{P}\right)_{3} \mathrm{RhCl}$ & $\mathrm{CH}_{2} \mathrm{Cl}_{2}$ & 100 & 0 & - & - & - & $-\mathrm{e}$ \\
\hline 4 & 13 & $\mathrm{Pd}(\mathrm{OH})_{2}$ & $\mathrm{AcOEt}$ & 100 & 89 & $100 / 0$ & $13 \mathbf{a}$ & 4.8 & - \\
\hline 5 & 15 & $\mathrm{Pd}(\mathrm{OH})_{2}$ & $\mathrm{AcOEt}$ & 1.5 & 82 & $100 / 0$ & $15 \mathbf{a}$ & 2.8 & $15 b(8 \%)$ \\
\hline 6 & 15 & $\mathrm{Pd}(\mathrm{OH})_{2}$ & $\mathrm{MeOH}$ & 1.5 & 95 & $100 / 0$ & $15 \mathbf{a}$ & 2.8 & - \\
\hline 7 & 8 & $\mathrm{Pd}(\mathrm{OH})_{2}$ & AcOEt & 1.5 & 72 & $100 / 0$ & $8 \mathbf{a}$ & 3.2 & $8 \mathbf{b}(8 \%)$ \\
\hline 8 & 14 & $\mathrm{Pd}(\mathrm{OH})_{2}$ & $\mathrm{AcOEt}$ & 1.5 & 76 & $100 / 0$ & $14 a$ & 3.7 & $\begin{array}{c}\text { 14b } \\
(10 \%)\end{array}$ \\
\hline 9 & 7 & $\mathrm{Pd}(\mathrm{OH})_{2}$ & $\mathrm{AcOEt}$ & 1.5 & 95 & $100 / 0$ & $7 a$ & 2.9 & - \\
\hline 10 & 9 & $\mathrm{Pd}(\mathrm{OH}) 2$ & $\mathrm{AcOEt}$ & 1.5 & 89 & $100 / 0$ & $9 \mathbf{a}$ & 3.4 & - \\
\hline 11 & 10 & $\mathrm{Pd}(\mathrm{OH}) 2$ & AcOEt & 1.5 & 55 & $80 / 20$ & $10 \mathbf{a}$ & - & $(25 \%)^{f}$ \\
\hline 12 & 11 & $\mathrm{Pd}(\mathrm{OH}) 2$ & AcOEt & 1.5 & 82 & $82 / 18$ & $11 a$ & - & - \\
\hline 13 & 12 & $\mathrm{Pd}(\mathrm{OH}) 2$ & AcOEt & 1.5 & 70 & $84 / 16$ & $12 \mathbf{a}$ & - & - \\
\hline 14 & 10 & $\left(\mathrm{Ph}_{3} \mathrm{P}\right)_{3} \mathrm{RhCl}$ & $\mathrm{CH}_{2} \mathrm{Cl}_{2}$ & 1.5 & 0 & - & - & - & - \\
\hline
\end{tabular}

(a) isolated yield. (b) determined by ${ }^{1} \mathrm{H}$ - and ${ }^{31} \mathrm{P}-\mathrm{NMR}$. (c) coupling constant of the $\alpha$ isomer. (d) no reaction. (e) $10 \%$ conversion of the starting exo-glycal into two unidentified side-products. (f) unidentified side-products.

\section{Diastereoselectivity of the hydrogenations}

The exoglycals bearing a bulky protective group at the 2-position (Piv or TBDMS, molecules 7, 8, 13, 14 and 15) gave exclusively the " $\alpha$ " $C$-galactosides. For each final ether, we performed NOESY experiments to confirm the "anomeric" configuration. In each case, a NOE effect 
between protons $\mathrm{H}-1$ and $\mathrm{H}-4$ was observed which corroborated with the configuration assigned from $J_{1-2}$ coupling constants. Whatever the steric hindrance of the group at the 3-position (H, Piv or TBDMS), the addition occurred always from the $\beta$ face specifically. For all these molecules, the " $\beta$ "-ether has never been observed. From this set of data, the steric hindrance at position 2 seems to be the only parameter governing the $\alpha$-specificity.

Then, we performed hydrogenations with a bulky pivaloate group at the 3-position and a less hindering functionality at the 2-position $(\mathrm{OH}, \mathrm{OCHO}, \mathrm{OAc}$, entries 11, 12 and 13). Surprisingly, $\alpha$-diastereoselectivities were observed in the three cases, the $\beta$-phosphonate being formed, at best, in 20\% yield. Exoglycal $\mathbf{1 0}$ gave a greater amount of side-products thus resulting in a poorer yield (entry 11). A better $\beta$-selectivity was expected with substrate $\mathbf{1 0}$ for two reasons: i) the steric hindrance of the $\mathrm{OH}$ group is rather small ii) $\mathrm{OH}$ groups have been shown to direct hydrogenations such a way that the $\mathrm{H}_{2}$ addition occurs from the same face of a cyclic system. Clearly, this directing effect does not apply with Pearlman catalyst. The directing effect of hydroxyl groups for the hydrogenation reaction has been often observed with rhodium and iridium catalysts. ${ }^{6}$ We thus used Crabtree's ${ }^{6}$ and Wilkinson's catalysts, under basic or non-basic conditions, using alcohols 10, 13 and 14 as substrates. Unfortunately, the expected phosphonates were never formed under these conditions (data not shown).

In conclusion, this study showed that the hydrogenation of exoglycals in the galactofuranose series, even unprotected at the 2-position, give a good to excellent $\alpha$-selectivity. Other parameters than the relative steric hindrance of the two faces of the glycal may be invoked to explain this result: owing to the abundant literature related to the stereoselectivity of reactions taking place at the anomeric center, it is quite reasonable to suggest that stereoelectronic or conformational effects may strongly contribute to the systematic $\alpha$-selectivity we observed. Conformationally, furanoses are usually less resticted than pyranoses. However, the presence of a trigonal $\mathrm{sp}^{2}$ hybrized anomeric carbon atom may favour a conformation placing the functional group in a position preventing the $\mathrm{H}_{2}$ addition from the $\alpha$-face. Moreover, the mechanism of the hydrogenation under heterogeneous catalysis being still poorly understood, we cannot rule out a directing effect of the two free doublets of the endocyclic oxygen, in other words, a stereoelectronic effect favoring the addition from the $\beta$-face of these exoglycals.

\section{Experimental Section}

General Procedures. All chemicals were purchased from Sigma, Aldrich or Fluka and were used without further purification. Tetrahydrofuran and toluene were freshly distilled over sodium benzophenone, dichloromethane over $\mathrm{P}_{2} \mathrm{O}_{5}$ and nitromethane over $\mathrm{CaH}_{2} .{ }^{1} \mathrm{H}-,{ }^{13} \mathrm{C}$ - and ${ }^{31} \mathrm{P}-\mathrm{NMR}$ spectra were recorded with Bruker AC-250 and AMX-400 spectrometers. All new compounds were characterized by ${ }^{1} \mathrm{H},{ }^{13} \mathrm{C},{ }^{31} \mathrm{P}-\mathrm{NMR}$ as well as by ${ }^{1} \mathrm{H}-{ }^{1} \mathrm{H}$ and ${ }^{1} \mathrm{H}-{ }^{13} \mathrm{C}$ correlation experiments. Specific optical rotations were measured on a Perkin Elmer 241 Polarimeter in a $1 \mathrm{dm}$ cell. Melting points were determined with a Büchi 535 apparatus. Purification by chromatography 
was performed on silica gel Kieselgel Si $60(40-63 \mu \mathrm{m})$ using cyclohexane (Cy) and AcOEt as eluent. Molecule 1 was prepared following literature data. ${ }^{10}$ Compounds 14, 15 and 15a were previously described. ${ }^{1,4}$

Atom and position numberings. We systematically numbered the phosphonate methylene group 1' and adopted the usual numbering for carbohydrates from 1 to 6 with 1 for the anomeric position.

\section{General procedure for exoglycal formation from lactones}

To a cooled $\left(-70^{\circ} \mathrm{C}\right)$ solution of dimethyl methylphosphonate (1 eq.) in anhydrous THF (18 mL) under Ar was added first butyl lithium (1 eq., $2.5 \mathrm{M}$ solution in hexane) and after $20 \mathrm{~min}$, a solution of lactone ( 0.4 eq.) in anhydrous THF. The temperature was maintained at $-70^{\circ} \mathrm{C}$ during $10 \mathrm{~min}$, the reaction mixture was then allowed to reach $-40^{\circ} \mathrm{C}$ over a $1 \mathrm{~h}$ period. The solution was then diluted with phosphate buffer $(1 \mathrm{M}, \mathrm{pH}=7)$ and extracted with $\mathrm{CH}_{2} \mathrm{Cl}_{2}$. The combined organic phases were dried over $\mathrm{MgSO}_{4}$, filtered and concentrated. The residue was dissolved in anhydrous $\mathrm{CH}_{2} \mathrm{Cl}_{2}$. At $0^{\circ} \mathrm{C}$ were added pyridine (10 eq.) and trifluoroacetic anhydride (5 eq.). After $3 \mathrm{~h}$ at $0^{\circ} \mathrm{C}$, the reaction was stopped by addition of saturated aqueous $\mathrm{NaHCO}_{3}$ followed by extraction with AcOEt. The organic phase was dried over $\mathrm{MgSO}_{4}$, filtered and concentrated at reduced pressure. The exoglycal was purified by chromatography on silica gel with cyclohexane/AcOEt (2:1). ${ }^{1,4}$

\section{General procedure for exoglycals hydrogenation}

To a solution of exoglycal in AcOEt (final concentration $0.15 \mathrm{M}$ ), was added wet $\mathrm{Pd}(\mathrm{OH})_{2}(0.4$ equiv./w) and the resulting suspension was degassed three times (the solution was left a few seconds under vacuum then under $\mathrm{H}_{2}$ atmosphere). The suspension was vigorously stirred under $\mathrm{H}_{2}$ (1.5 bar) for 24 hours, filtrated over celite and concentrated under vacuum.

2-O-tert-Butyldimethylsilyl-5,6-O-(dimethyl-methylidene)-D-galactono-1,4-lactone (2). A suspension of lactone $\mathbf{1}^{10}$ (890 mg, $4.08 \mathrm{mmol}$ ), TBDMSCl (1.65 g, $4.28 \mathrm{mmol}, 1.05$ eq.) and imidazole (415 mg, $6.11 \mathrm{mmol}, 1.5$ eq.) in anhydrous DMF (12 mL) was stirred, under argon, one hour at $0^{\circ} \mathrm{C}$, then overnight at room temperature. The solvent was removed and the resulting mixture was suspended in ether $(15 \mathrm{~mL})$ and washed twice with water $(10 \mathrm{~mL})$. The organic layer was dried over $\mathrm{MgSO}_{4}$, filtered and concentrated. The resulting crude reaction mixture was submitted to purification by chromatography on silica gel (Cy/AcOEt : 3/1) to give 2 (880 mg, $85 \%$ ) as a white solid. $[\alpha]_{\mathrm{D}}{ }^{23}-7.1$ (c 1.0, $\left.\mathrm{CHCl}_{3}\right)$; m.p. $118^{\circ} \mathrm{C} ;{ }^{1} \mathrm{H}-\mathrm{NMR}\left(400 \mathrm{MHz}, \mathrm{CDCl}_{3}\right) \delta$ $4.42\left(\mathrm{~d}, J_{2-3}=8.5 \mathrm{~Hz}, 1 \mathrm{H}, \mathrm{H}-2\right), 4.39\left(\mathrm{td}, J_{4-5}=3.9 \mathrm{~Hz}, J_{5-6 \mathrm{a}, \mathrm{b}}=6.8 \mathrm{~Hz}, 1 \mathrm{H}, \mathrm{H}-5\right), 4.32\left(\mathrm{dd}, J_{2-3}=\right.$ $\left.8.5 \mathrm{~Hz}, J_{3-4}=8.1 \mathrm{~Hz}, 1 \mathrm{H}, \mathrm{H}-3\right), 4.13\left(\mathrm{ABX}, J_{5-6 \mathrm{a}}=6.8 \mathrm{~Hz}, J_{6 \mathrm{a}-6 \mathrm{~b}}=8.7 \mathrm{~Hz}, 1 \mathrm{H}, \mathrm{H}-6 \mathrm{a}\right), 4.10$ (dd, $\left.J_{3-4}=8.1 \mathrm{~Hz}, J_{4-5}=3.9 \mathrm{~Hz}, 1 \mathrm{H}, \mathrm{H}-4\right), 4.00\left(\mathrm{ABX}, J_{5-6 \mathrm{~b}}=6.8 \mathrm{~Hz}, J_{6 \mathrm{a}-6 \mathrm{~b}}=8.7 \mathrm{~Hz}, 1 \mathrm{H}, \mathrm{H}-6 \mathrm{~b}\right), 1.44$, 1.40 (2s, 6H, $\mathrm{C}\left(\mathrm{CH}_{3}\right)_{2}$, acetonide), 0.96 (s, 9H, Si-tBu), 0.16 (s, 3H, Si-Me), 0.13 (s, 3H, Si-Me); ${ }^{13} \mathrm{C}-\mathrm{NMR}\left(100 \mathrm{MHz}, \mathrm{CDCl}_{3}\right) \delta 172.57(\mathrm{C}-1), 110.16\left(\mathrm{C}\left(\mathrm{CH}_{3}\right)_{2}\right.$, acetonide), $78.35(\mathrm{C}-4), 75.56$ 
(C-2), 74.90 (C-3), $73.73(\mathrm{C}-5), 64.84(\mathrm{C}-6), 26.06\left(\mathrm{C}\left(\mathrm{CH}_{3}\right)_{2}\right.$, acetonide), $25.58\left(\mathrm{Si}-\mathrm{C}\left(\mathrm{CH}_{3}\right)_{3}\right)$,

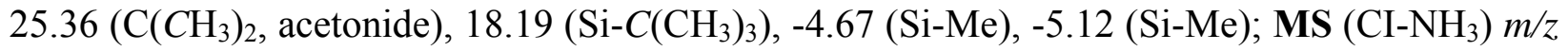
$350\left[\mathrm{M}+\mathrm{NH}_{4}\right]^{+}$; El. Anal. for $\mathrm{C}_{15} \mathrm{H}_{28} \mathrm{O}_{6} \mathrm{Si}$ : calc. (\%) C 54.19 H 8.19. mes. C 54.04 H 8.67.

\section{2-O-tert-Butyldimethylsilyl-5,6-O-(dimethyl-methylidene)-3-O-pivaloyl-D-galactono-1,4-}

lactone (3). To a suspension of lactone $2(1.0 \mathrm{~g}, 3.0 \mathrm{mmol})$ in anhydrous acetonitrile $(20 \mathrm{~mL})$ were added pyridine $(5 \mathrm{~mL})$ and pivaloyl chloride $(0.74 \mathrm{~mL}, 6 \mathrm{mmol}, 2$ eq. $)$. The mixture was then stirred overnight at $80^{\circ} \mathrm{C}$, concentrated and dried under vacuum. The resulting crude mixture was chromatographed on silica gel (Cy/AcOEt : 4/1) to give $3(2.79 \mathrm{~g}, 93 \%)$ as a white solid. $[\alpha]_{\mathrm{D}}{ }^{23}+12.1\left(\mathrm{c} 1.0, \mathrm{CHCl}_{3}\right)$; m.p. $69-70^{\circ} \mathrm{C} ;{ }^{1} \mathrm{H}-\mathrm{NMR}\left(400 \mathrm{MHz}, \mathrm{CDCl}_{3}\right) \delta 5.34\left(\mathrm{t} J_{2-3}=J_{3-4}\right.$ $=5.7 \mathrm{~Hz}, 1 \mathrm{H}, \mathrm{H}-3), 4.52\left(\mathrm{~d}, J_{2-3}=5.7 \mathrm{~Hz}, 1 \mathrm{H}, \mathrm{H}-2\right), 4.43\left(\mathrm{td}, J_{4-5}=3.9 \mathrm{~Hz}, J_{5-6 \mathrm{a}, \mathrm{b}}=6.6 \mathrm{~Hz}, 1 \mathrm{H}\right.$, $\mathrm{H}-5), 4.20\left(\mathrm{dd}, J_{3-4}=5.7 \mathrm{~Hz}, J_{4-5}=3.9 \mathrm{~Hz}, 1 \mathrm{H}, \mathrm{H}-4\right), 4.11\left(\mathrm{ABX}, J_{5-6 \mathrm{a}}=6.6 \mathrm{~Hz}, J_{6 \mathrm{a}-6 \mathrm{~b}}=8.4 \mathrm{~Hz}\right.$, $1 \mathrm{H}, \mathrm{H}-6 \mathrm{a}), 3.94\left(\mathrm{ABX}, J_{5-6 \mathrm{~b}}=6.6 \mathrm{~Hz}, J_{6 \mathrm{a}-6 \mathrm{~b}}=8.4 \mathrm{~Hz}, 1 \mathrm{H}, \mathrm{H}-6 \mathrm{~b}\right), 1.45,1.37\left(2 \mathrm{~s}, 6 \mathrm{H}, \mathrm{C}\left(\mathrm{CH}_{3}\right)_{2}\right.$, acetonide), 1.26 (s, 9H, Piv), 0.92 (s, 9H, Si-tBu), 0.16 (s, 3H, Si-Me), 0.13 (s, 3H, Si-Me); ${ }^{13} \mathrm{C}-$

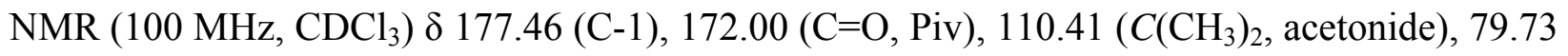
(C-4), 76.17 (C-3), 74.77 (C-5), 73.17 (C-2), 65.04 (C-6), $38.67\left(\mathrm{CO}-\mathrm{C}\left(\mathrm{CH}_{3}\right)_{3}\right.$, Piv), 27.03 (CO-

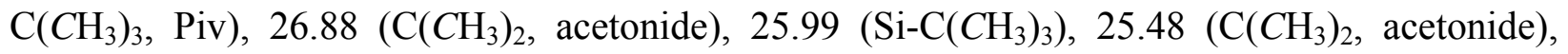
$17.79\left(\mathrm{Si}-\mathrm{C}\left(\mathrm{CH}_{3}\right)_{3}\right),-4.66(\mathrm{Si}-\mathrm{Me}),-5.39(\mathrm{Si}-\mathrm{Me})$; $\mathrm{MS}\left(\mathrm{CI}-\mathrm{NH}_{3}\right) \mathrm{m} / \mathrm{z} 434\left[\mathrm{M}+\mathrm{NH}_{4}\right]^{+}$; El. Anal. for $\mathrm{C}_{20} \mathrm{H}_{36} \mathrm{O}_{7} \mathrm{Si}$ : calc. (\%) C $57.66 \mathrm{H}$ 8.71. mes. C $57.57 \mathrm{H} 8.85$.

2,5,6-Tri-O-tert-butyldimethylsilyl-3-O-pivaloyl-D-galactono-1,4-lactone (6). A solution of lactone $3(500 \mathrm{mg}, 1.2 \mathrm{mmol})$ in $\mathrm{AcOH} / \mathrm{H}_{2} \mathrm{O} 4 / 1(9.0 \mathrm{~mL})$ was stirred at $80^{\circ} \mathrm{C}$ for 3 hours. After concentration under vacuum, the resulting oil was submitted three times to azeotropic distillation with toluene and dried overnight under vacuum before being dissolved in anhydrous DMF (7 $\mathrm{mL})$. To this solution were added TBDMSCl (1.2 g, $8 \mathrm{mmol}, 6.7$ eq. $)$ and imidazole $(0.81 \mathrm{~g}, 11.9$ mmol, 10 eq.). This mixture was then heated 5 hours at $60^{\circ} \mathrm{C}$. The solvent was removed under reduced pressure and the resulting mixture was suspended in ether $(15 \mathrm{~mL})$ and washed twice with water $(10 \mathrm{~mL})$. The organic phase was dried over $\mathrm{MgSO}_{4}$, filtered and concentrated. The crude reaction mixture was purified by chromatography on silica gel (Cy/AcOEt : 35/1) to give 6 (536 mg, $74 \%$ ) as a colourless oil. $[\alpha]_{\mathrm{D}}{ }^{23}-10.4\left(\mathrm{c} 0.9, \mathrm{CHCl}_{3}\right)$; m.p. $52-53{ }^{\circ} \mathrm{C} ;{ }^{1} \mathrm{H}-\mathrm{NMR}(400$ $\left.\mathrm{MHz} \mathrm{CDCl}_{3}\right) \delta 5.41\left(\mathrm{t}, J_{2-3}=J_{3-4}=5.6 \mathrm{~Hz}, 1 \mathrm{H}, \mathrm{H}-3\right), 4.57\left(\mathrm{~d}, J_{2-3}=5.6 \mathrm{~Hz}, 1 \mathrm{H}, \mathrm{H}-2\right), 4.41(\mathrm{dd}$, $\left.J_{3-4}=5.6 \mathrm{~Hz} J_{4-5}=1.6 \mathrm{~Hz}, 1 \mathrm{H}, \mathrm{H}-4\right), 3.97\left(\mathrm{ddd}, J_{4-5}=1.6 \mathrm{~Hz}, J_{5-6 \mathrm{a}}=8.9 \mathrm{~Hz}, J_{5-6 \mathrm{~b}}=5.4 \mathrm{~Hz}, 1 \mathrm{H}\right.$, $\mathrm{H}-5), 3.66\left(\mathrm{ABX}, J_{5-6 \mathrm{a}}=8.9 \mathrm{~Hz}, J_{6 \mathrm{a}-6 \mathrm{~b}}=9.8 \mathrm{~Hz}, 1 \mathrm{H}, \mathrm{H}-6 \mathrm{a}\right), 3.59\left(\mathrm{ABX}, J_{5-6 \mathrm{~b}}=5.4 \mathrm{~Hz}, J_{6 \mathrm{a}-6 \mathrm{~b}}=9.8\right.$ $\mathrm{Hz}, 1 \mathrm{H}, \mathrm{H}-6 \mathrm{~b}), 1.25$ (s, 9H, Piv), 0.93 (s, 9H, Si-tBu), 0.90 (s, 9H, Si-tBu), 0.89 (s, 9H, Si-tBu), 0.20 (s, 3H, Si-Me), 0.19 (s, 3H, Si-Me), 0.16 (s, 3H, Si-Me), 0.12 (s, 3H, Si-Me), 0.08 (s, 3H, Si-Me), 0.07 (s, 3H, Si-Me); ${ }^{13} \mathrm{C}-\mathrm{NMR}\left(100 \mathrm{MHz}, \mathrm{CDCl}_{3}\right) \delta 177.52$ (C=O, Piv), 172.58 (C-1), 79.90 (C-4), 76.47 (C-3), 73.56 (C-2), 71.99 (C-5), 62.51 (C-6), 38.58 (CO-C(CH$\left.)_{3}, \mathrm{Piv}\right), 27.02$ $\left(\mathrm{CO}-\mathrm{C}\left(\mathrm{CH}_{3}\right)_{3}, \mathrm{Piv}\right), 25.76\left(\mathrm{Si}-\mathrm{C}\left(\mathrm{CH}_{3}\right)_{3}\right), 25.72\left(\mathrm{Si}-\mathrm{C}\left(\mathrm{CH}_{3}\right)_{3}\right), 25.51\left(\mathrm{Si}-\mathrm{C}\left(\mathrm{CH}_{3}\right)_{3}\right)$, 18.11 (Si$\left.\mathrm{C}\left(\mathrm{CH}_{3}\right)_{3}\right), 18.08\left(\mathrm{Si}-\mathrm{C}\left(\mathrm{CH}_{3}\right)_{3}\right), 17.92\left(\mathrm{Si}-\mathrm{C}\left(\mathrm{CH}_{3}\right)_{3}\right),-4.11(\mathrm{Si}-\mathrm{Me}),-4.71(\mathrm{Si}-\mathrm{Me}),-4.90(\mathrm{Si}-\mathrm{Me})$, 5.39 (Si-Me), -5.48 (Si-Me), -5.51 (Si-Me); MS (CI-NH $)_{3} \mathrm{~m} / \mathrm{z} 622\left[\mathrm{M}+\mathrm{NH}_{4}\right]^{+}$; El. Anal. for $\mathrm{C}_{29} \mathrm{H}_{60} \mathrm{O}_{7} \mathrm{Si}_{3}$ : calc. (\%) C $57.57 \mathrm{H}$ 9.99. mes. C $57.50 \mathrm{H} 10.02$. 


\section{(1(1')Z)-2-O-tert-Butyldimethylsilyl-1-(dimethoxyphosphoryl)}

methylidene-5,6-O(dimethyl-ethylidene)-3-O-pivaloyl-D-galactofuranose (7). The product $\mathbf{6}$ was prepared according to the general procedure for exoglycal formation from lactones. After chromatography on silica gel (Cy/AcOEt : 1/2), compound $7(72 \%)$ was isolated as a white solid. $[\alpha]_{\mathrm{D}}{ }^{21}+28.6(\mathrm{c}$ 0.9, $\mathrm{CHCl}_{3}$ ); m.p. $49-50^{\circ} \mathrm{C}$; ${ }^{1} \mathrm{H}-\mathrm{NMR}\left(400 \mathrm{MHz}, \mathrm{CDCl}_{3}\right) \delta 5.13\left(\mathrm{t}, J_{2-3}=J_{3-4}=4.5 \mathrm{~Hz}, 1 \mathrm{H}, \mathrm{H}-3\right)$, $4.70\left(\mathrm{td}, J_{1^{\prime}-2}=1.3 \mathrm{~Hz}, J_{2-\mathrm{P}}=J_{2-3}=4.5 \mathrm{~Hz}, 1 \mathrm{H}, \mathrm{H}-2\right), 4.62\left(\mathrm{dd}, J_{1^{\prime}-2}=1.3 \mathrm{~Hz}, J_{1^{\prime}-\mathrm{P}}=9.4 \mathrm{~Hz}, 1 \mathrm{H}\right.$, H-1'), $4.41\left(\mathrm{td}, J_{4-5}=4.5 \mathrm{~Hz}, J_{5-6}=6.8 \mathrm{~Hz}, 1 \mathrm{H}, \mathrm{H}-5\right), 4.30\left(\mathrm{t}, J_{3-4}=J_{4-5}=4.5 \mathrm{~Hz}, 1 \mathrm{H}, \mathrm{H}-4\right), 4.06$ $\left(\mathrm{ABX}, J_{5-6 \mathrm{a}}=6.8 \mathrm{~Hz}, J_{6 \mathrm{a}-6 \mathrm{~b}}=8.3 \mathrm{~Hz}, 1 \mathrm{H}, \mathrm{H}-6 \mathrm{a}\right), 3.98\left(\mathrm{ABX}, J_{5-6 \mathrm{~b}}=6.8 \mathrm{~Hz}, J_{6 \mathrm{a}-6 \mathrm{~b}}=8.3 \mathrm{~Hz}, 1 \mathrm{H}\right.$, $\mathrm{H}-6 \mathrm{~b}), 3.77$ (d, $\left.J_{\mathrm{H}-\mathrm{P}}=11.3 \mathrm{~Hz}, 3 \mathrm{H}, \mathrm{OMe}\right), 3.75$ (d, $\left.J_{\mathrm{H}-\mathrm{P}}=11.4 \mathrm{~Hz}, 3 \mathrm{H}, \mathrm{OMe}\right), 1.45,1.37(2 \mathrm{~s}, 6 \mathrm{H}$, $\mathrm{C}\left(\mathrm{CH}_{3}\right)_{2}$, acetonide), 1.23 (s, 9H, Piv), 0.92 (s, 9H, Si-tBu), 0.16 (s, 3H, Si-Me), 0.13 (s, 3H, Si$\mathrm{Me}$ ); ${ }^{13} \mathrm{C}-\mathrm{NMR}\left(100 \mathrm{MHz}, \mathrm{CDCl}_{3}\right) \delta 177.53$ (C=O, Piv), 171.30 (d, $\left.J_{1-\mathrm{P}}=2.3 \mathrm{~Hz}, \mathrm{C}-1\right), 109.99$ $\left(C\left(\mathrm{CH}_{3}\right)_{2}\right.$, acetonide), $85.64(\mathrm{C}-4), 82.84\left(\mathrm{~d}, J_{1}{ }^{\prime}-\mathrm{P}=196.1 \mathrm{~Hz}, \mathrm{C}-1^{\prime}\right), 77.43(\mathrm{C}-3), 77.10\left(\mathrm{~d}, J_{2-\mathrm{P}}=\right.$ $14.0 \mathrm{~Hz}, \mathrm{C}-2), 74.77$ (C-5), 64.96 (C-6), 52.40 (d, $\left.J_{\mathrm{C}-\mathrm{P}}=5.7 \mathrm{~Hz}, \mathrm{OMe}\right), 52.10$ (d, $J_{\mathrm{C}-\mathrm{P}}=5.6 \mathrm{~Hz}$,

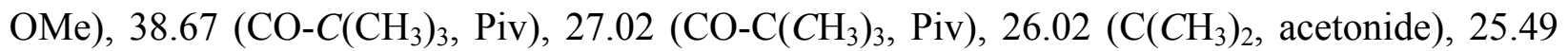
$\left(\mathrm{Si}-\mathrm{C}\left(\mathrm{CH}_{3}\right)_{3}\right), 25.24\left(\mathrm{C}\left(\mathrm{CH}_{3}\right)_{2}\right.$, acetonide), $17.79\left(\mathrm{Si}-\mathrm{C}\left(\mathrm{CH}_{3}\right)_{3}\right),-4.74(\mathrm{Si}-\mathrm{Me}),-4.78(\mathrm{Si}-\mathrm{Me}){ }^{31} \mathrm{P}-$ NMR $\left(101 \mathrm{MHz}, \mathrm{CDCl}_{3}\right) \delta$ 20.49; MS $\left(\mathrm{CI}_{-\mathrm{NH}_{3}}\right) \mathrm{m} / \mathrm{z} 523[\mathrm{M}+\mathrm{H}]^{+}$; El. Anal. for $\mathrm{C}_{23} \mathrm{H}_{43} \mathrm{O}_{9} \mathrm{PSi}$ : calc. (\%) C 52.86 H 8.29. mes. C 52.53 H 8.56.

Dimethyl (2-O-tert-butyldimethylsilyl-5,6-O-(dimethyl-methylidene)-3-O-pivaloyl-a-Dgalactofuranosyl) methanephosphonate (7a). The "general procedure for exoglycals hydrogenation" was applied. After chromatography on silica gel (AcOEt), compound 7a (95\%) was isolated as a colourless oil. $[\alpha]_{\mathrm{D}}{ }^{19}-9.5$ (c $\left.0.77, \mathrm{CHCl}_{3}\right) ;{ }^{1} \mathrm{H}-\mathrm{NMR}\left(400 \mathrm{MHz}, \mathrm{CDCl}_{3}\right) \delta 4.79$ $\left(\mathrm{d}, J_{3-4}=2.2 \mathrm{~Hz}, 1 \mathrm{H}, \mathrm{H}-3\right), 4.31\left(\mathrm{q}, J_{4-5}=6.8 \mathrm{~Hz}, 1 \mathrm{H}, \mathrm{H}-5\right), 4.22\left(\mathrm{tdd}, J_{1-2}=2.9 \mathrm{~Hz}, J_{1}{ }^{\prime}\right.$ a,b-1 $=6.4$ $\left.\mathrm{Hz}, J_{1-\mathrm{P}}=11.4 \mathrm{~Hz}, 1 \mathrm{H}, \mathrm{H}-1\right), 4.00\left(\mathrm{ABX}, J_{5-6 \mathrm{a}}=6.8 \mathrm{~Hz}, J_{6 \mathrm{a}-6 \mathrm{~b}}=8.6 \mathrm{~Hz}, 1 \mathrm{H}, \mathrm{H}-6 \mathrm{a}\right), 3.96\left(\mathrm{~d}, J_{1-2}=\right.$ $2.9 \mathrm{~Hz}, 1 \mathrm{H}, \mathrm{H}-2), 3.88\left(\mathrm{ABX}, J_{5-6 \mathrm{~b}}=6.1 \mathrm{~Hz}, J_{6 \mathrm{a}-6 \mathrm{~b}}=8.6 \mathrm{~Hz}, 1 \mathrm{H}, \mathrm{H}-6 \mathrm{~b}\right), 3.83$ (dd, $J_{3-4}=2.2 \mathrm{~Hz}$, $\left.J_{4-5}=6.8 \mathrm{~Hz}, 1 \mathrm{H}, \mathrm{H}-4\right), 3.76\left(\mathrm{~d}, J_{\mathrm{H}-\mathrm{P}}=11.3 \mathrm{~Hz}, 3 \mathrm{H}, \mathrm{OMe}\right), 3.73\left(\mathrm{~d}, J_{\mathrm{H}-\mathrm{P}}=11.3 \mathrm{~Hz}, 3 \mathrm{H}, \mathrm{OMe}\right)$, $2.23\left(\mathrm{ABXX}, J_{1}\right.$ 'a-1'b $=15.3 \mathrm{~Hz}, J_{1}$ 'a-1 $=6.4 \mathrm{~Hz}, J_{1}$ 'a-P $=18.2 \mathrm{~Hz}, 1 \mathrm{H}, \mathrm{H}-1$ 'a), 2.15 (ABXX', $J_{1}$ 'a$1^{\prime} \mathrm{b}=15.3 \mathrm{~Hz}, J_{1}{ }^{\prime} \mathrm{b}-1=6.4 \mathrm{~Hz}, J_{1}{ }^{\prime} \mathrm{b}-\mathrm{P}=18.3 \mathrm{~Hz}, 1 \mathrm{H}, \mathrm{H}-1$ 'b $), 1.40,1.33\left(2 \mathrm{~s}, 6 \mathrm{H}, \mathrm{C}\left(\mathrm{CH}_{3}\right)_{2}\right.$, acetonide), 1.19 (s, 9H, Piv), 0.91 (s, 9H, Si-tBu), 0.17 (s, 3H, Si-Me), 0.11 (s, 3H, Si-Me); ${ }^{13} \mathrm{C}-$ NMR $\left(100 \mathrm{MHz}, \mathrm{CDCl}_{3}\right) \delta 177.30(\mathrm{C}=\mathrm{O}, \mathrm{Piv}), 109.64\left(\mathrm{C}\left(\mathrm{CH}_{3}\right)_{2}\right.$, acetonide), $84.50(\mathrm{C}-4), 79.82$ (C-3), 76.92 (C-1), 76.90 (d, $\left.J_{2-\mathrm{P}}=8.4 \mathrm{~Hz}, \mathrm{C}-2\right), 75.60$ (C-5), 65.40 (C-6), 52.57 (d, $J_{\mathrm{C}-\mathrm{P}}=6.5 \mathrm{~Hz}$,

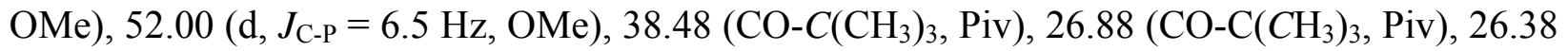
$\left(\mathrm{C}\left(\mathrm{CH}_{3}\right)_{2}\right.$, acetonide), $25.66\left(\mathrm{Si}-\mathrm{C}\left(\mathrm{CH}_{3}\right)_{3}\right), 24.88\left(\mathrm{C}\left(\mathrm{CH}_{3}\right)_{2}\right.$, acetonide $), 24.71\left(\mathrm{~d}, J_{1}\right.$ '- $\mathrm{P}=141.6 \mathrm{~Hz}$, C-1'), $17.95\left(\mathrm{Si}-\mathrm{C}\left(\mathrm{CH}_{3}\right)_{3}\right),-4.64(\mathrm{Si}-\mathrm{Me}),-5.61$ (Si-Me); ${ }^{31} \mathrm{P}-\mathrm{NMR}\left(101 \mathrm{MHz}, \mathrm{CDCl}_{3}\right) \delta 31.33$; MS $\left(\mathrm{CI}-\mathrm{NH}_{3}\right) \mathrm{m} / \mathrm{z} 525(25 \%)[\mathrm{M}+\mathrm{H}]^{+}, 542(100 \%)\left[\mathrm{M}+\mathrm{NH}_{4}\right]^{+}$; El. Anal. for $\mathrm{C}_{23} \mathrm{H}_{45} \mathrm{O}_{9} \mathrm{PSi}$ : calc. (\%) C 52.65 H 8.65. mes. C $52.51 \mathrm{H} 8.82$.

(1(1')Z)-2,5,6-Tri-O-tert-butyldimethylsilyl-1-(dimethoxyphosphoryl)methylidene-3-O-

pivaloyl-D-galactofuranose (8). Product 8 was prepared according to the general procedure for exoglycal formation from lactones. After chromatography on silica gel (Cy/AcOEt: 3/1), compound $8(76 \%)$ was isolated as a white solid. $[\alpha]_{\mathrm{D}}{ }^{22}+8.15\left(\mathrm{c} 1.2, \mathrm{CHCl}_{3}\right)$; m.p. $61{ }^{\circ} \mathrm{C} ;{ }^{1} \mathrm{H}-$ $\operatorname{NMR}\left(400 \mathrm{MHz}, \mathrm{CDCl}_{3}\right) \delta 5.25\left(\mathrm{t}, J_{2-3}=J_{3-4}=5.0 \mathrm{~Hz}, 1 \mathrm{H}, \mathrm{H}-3\right), 4.85\left(\mathrm{ddd}, J_{1^{\prime}-2}=1.6 \mathrm{~Hz}, J_{2-3}=\right.$ 
$\left.5.0 \mathrm{~Hz}, J_{2-\mathrm{P}}=4.1 \mathrm{~Hz}, 1 \mathrm{H}, \mathrm{H}-2\right), 4.53\left(\mathrm{dd}, J_{1^{\prime}-2}=1.6 \mathrm{~Hz}, J_{1}{ }^{\prime}-\mathrm{P}=10.1 \mathrm{~Hz}, 1 \mathrm{H}, \mathrm{H}-1^{\prime}\right.$ '), $4.41\left(\mathrm{dd}, J_{3-4}\right.$ $\left.=5.0 \mathrm{~Hz}, J_{4-5}=1.8 \mathrm{~Hz}, 1 \mathrm{H}, \mathrm{H}-4\right), 4.01\left(\mathrm{ddd}, J_{4-5}=1.8 \mathrm{~Hz}, J_{5-6 \mathrm{a}}=8.8 \mathrm{~Hz}, J_{5-6 \mathrm{~b}}=5.3 \mathrm{~Hz}, 1 \mathrm{H}, \mathrm{H}-5\right)$, $3.72\left(\mathrm{ABX}, J_{5-6 \mathrm{a}}=8.8 \mathrm{~Hz}, J_{6 \mathrm{a}-6 \mathrm{~b}}=9.6 \mathrm{~Hz}, 1 \mathrm{H}, \mathrm{H}-6 \mathrm{a}\right), 3.72\left(\mathrm{~d}, J_{\mathrm{H}-\mathrm{P}}=11.3 \mathrm{~Hz}, 3 \mathrm{H}, \mathrm{OMe}\right), 3.70(\mathrm{~d}$, $\left.J_{\mathrm{H}-\mathrm{P}}=11.5 \mathrm{~Hz}, 3 \mathrm{H}, \mathrm{OMe}\right), 3.58\left(\mathrm{ABX}, J_{5-6 \mathrm{~b}}=5.3 \mathrm{~Hz}, J_{6 \mathrm{a}-6 \mathrm{~b}}=9.6 \mathrm{~Hz}, 1 \mathrm{H}, \mathrm{H}-6 \mathrm{~b}\right), 1.21(\mathrm{~s}, 9 \mathrm{H}$, Piv), 0.90 (s, 9H, Si-tBu), 0.87 (s, 9H, Si-tBu), 0.86 (s, 9H, Si-tBu), 0.17 (s, 3H, Si-Me), 0.13 (s, 3H, Si-Me), 0.08 (s, 6H, Si-Me), 0.06 (s, 3H, Si-Me), 0.05 (s, 3H, Si-Me); ${ }^{13} \mathrm{C}-\mathrm{NMR}$ (100 MHz, $\left.\mathrm{CDCl}_{3}\right) \delta 177.71(\mathrm{C}=\mathrm{O}, \mathrm{Piv}), 172.10\left(\mathrm{~d}, J_{1-\mathrm{P}}=2.1 \mathrm{~Hz}, \mathrm{C}-1\right), 85.77$ (C-4), 80.34 (d, $J_{1}$ '-P $=195.8$ Hz, C-1'), 77.77 (C-3), 77.34 (d, J2-P $=13.9$ Hz, C-2), 72.78 (C-5), 62.65 (C-6), 52.16 (d, $J_{\mathrm{C}-\mathrm{P}}=$ $5.6 \mathrm{~Hz}, \mathrm{OMe}), 52.00\left(\mathrm{~d}, J_{\mathrm{C}-\mathrm{P}}=5.2 \mathrm{~Hz}, \mathrm{OMe}\right), 38.52\left(\mathrm{CO}-\mathrm{C}\left(\mathrm{CH}_{3}\right)_{3}, \mathrm{Piv}\right), 27.02\left(\mathrm{CO}-\mathrm{C}\left(\mathrm{CH}_{3}\right)_{3}\right.$, Piv), $25.72\left(2 \mathrm{Si}-\mathrm{C}\left(\mathrm{CH}_{3}\right)_{3}\right), 25.53\left(\mathrm{Si}-\mathrm{C}\left(\mathrm{CH}_{3}\right)_{3}\right), 18.02\left(\mathrm{Si}-\mathrm{C}\left(\mathrm{CH}_{3}\right)_{3}\right), 17.91\left(\mathrm{Si}-\mathrm{C}\left(\mathrm{CH}_{3}\right)_{3}\right), 17.78$ $\left(\mathrm{Si}-\mathrm{C}\left(\mathrm{CH}_{3}\right)_{3}\right),-4.16(\mathrm{Si}-\mathrm{Me}),-4.70(\mathrm{Si}-\mathrm{Me}),-4.99$ (Si-Me), -5.08 (Si-Me), -5.54 (Si-Me), -5.62 $(\mathrm{Si}-\mathrm{Me}) ;{ }^{31} \mathrm{P}-\mathrm{NMR}\left(101 \mathrm{MHz}, \mathrm{CDCl}_{3}\right) \delta 21.85 ; \mathrm{MS}\left(\mathrm{CI}-\mathrm{NH}_{3}\right) \mathrm{m} / \mathrm{z} 711(100 \%)[\mathrm{M}+\mathrm{H}]^{+}, 728$ $(80 \%)\left[\mathrm{M}+\mathrm{NH}_{4}\right]^{+}$; El. Anal. for $\mathrm{C}_{32} \mathrm{H}_{67} \mathrm{O}_{9} \mathrm{PSi}_{3}$ : calc. (\%) C 54.05 H 9.50. mes. C 53.93 H 9.63.

Dimethyl (2,5,6-tri-O-tert-butyldimethylsilyl-3-O-pivaloyl- $\alpha$-D-galactofuranosyl) methanephosphonate (8). The "general procedure for exoglycals hydrogenation" was applied. After chromatography on silica gel (Cy/AcOEt : 2/1), compound 8a (72\%) and $\mathbf{8 b}(8 \%)$ were isolated. $[\alpha]_{\mathrm{D}}{ }^{21}-7.1\left(\mathrm{c} 0.6, \mathrm{CHCl}_{3}\right) ;{ }^{1} \mathrm{H}-\mathrm{NMR}\left(400 \mathrm{MHz}, \mathrm{CDCl}_{3}\right) \delta 5.08\left(\mathrm{dd}, J_{2-3}=1.3 \mathrm{~Hz}, J_{3-4}=\right.$ $2.4 \mathrm{~Hz}, 1 \mathrm{H}, \mathrm{H}-3), 4.32\left(\mathrm{tdd}, J_{1-2}=3.2 \mathrm{~Hz}, J_{1}{ }^{\prime} \mathrm{a}-1=6.9 \mathrm{~Hz}, J_{1^{\prime} \mathrm{b}-1}=5.6 \mathrm{~Hz}, J_{1-\mathrm{P}}=9.8 \mathrm{~Hz}, 1 \mathrm{H}, \mathrm{H}-1\right)$, $3.93\left(\mathrm{dd}, J_{3-4}=2.4 \mathrm{~Hz}, J_{4-5}=7.1 \mathrm{~Hz}, 1 \mathrm{H}, \mathrm{H}-4\right), 3.91$ (d, $\left.J_{2-\mathrm{P}}=9.8 \mathrm{~Hz}, 1 \mathrm{H}, \mathrm{H}-2\right), 3.87$ (ddd, $J_{4-5}=$ $\left.7.1 \mathrm{~Hz}, J_{5-6 \mathrm{a}}=4.1 \mathrm{~Hz}, J_{5-6 \mathrm{~b}}=5.4 \mathrm{~Hz}, 1 \mathrm{H}, \mathrm{H}-5\right), 3.79\left(\mathrm{~d}, J_{\mathrm{H}-\mathrm{P}}=11.0 \mathrm{~Hz}, 3 \mathrm{H}, \mathrm{OMe}\right), 3.77\left(\mathrm{~d}, J_{\mathrm{H}-\mathrm{P}}=\right.$ $11.0 \mathrm{~Hz}, 3 \mathrm{H}, \mathrm{OMe}), 3.72\left(\mathrm{ABX}, J_{5-6 \mathrm{a}}=4.1 \mathrm{~Hz}, J_{6 \mathrm{a}-6 \mathrm{~b}}=10.6 \mathrm{~Hz}, 1 \mathrm{H}, \mathrm{H}-6 \mathrm{a}\right), 3.59$ (ABX, $J_{5-6 \mathrm{~b}}=$

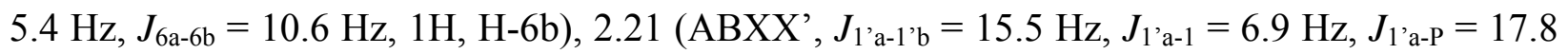

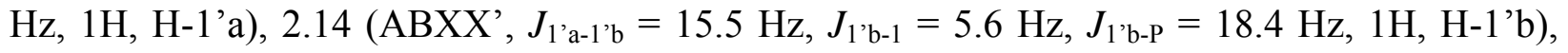
1.21 (s, 9H, Piv), 0.95 (s, 9H, Si-tBu), 0.91 (s, 9H, Si-tBu), 0.90 (s, 9H, Si-tBu), 0.23 (s, 3H, SiMe), 0.13 (s, 3H, Si-Me), 0.11 (s, 3H, Si-Me), 0.10 (s, 3H, Si-Me), 0.06 (s, 3H, Si-Me), 0.05 (s, 3H, Si-Me); ${ }^{13} \mathrm{C}-\mathrm{NMR}\left(100 \mathrm{MHz}, \mathrm{CDCl}_{3}\right) \delta 177.20$ (C=O, Piv), 84.38 (C-4), 79.21 (C-3), 77.29 $\left(\mathrm{d}, J_{2-\mathrm{P}}=9.8 \mathrm{~Hz}, \mathrm{C}-2\right), 75.26\left(\mathrm{~d}, J_{1-\mathrm{P}}=0.9 \mathrm{~Hz}, \mathrm{C}-1\right), 73.66(\mathrm{C}-5), 65.64(\mathrm{C}-6), 52.54\left(\mathrm{~d}, J_{\mathrm{C}-\mathrm{P}}=6.4\right.$ $\mathrm{Hz}, \mathrm{OMe}), 52.15\left(\mathrm{~d}, J_{\mathrm{C}-\mathrm{P}}=6.5 \mathrm{~Hz}, \mathrm{OMe}\right), 38.4\left(\mathrm{CO}-\mathrm{C}\left(\mathrm{CH}_{3}\right)_{3}, \mathrm{Piv}\right), 27.05\left(\mathrm{CO}-\mathrm{C}\left(\mathrm{CH}_{3}\right)_{3}, \mathrm{Piv}\right)$, $25.98\left(\mathrm{Si}-\mathrm{C}\left(\mathrm{CH}_{3}\right)_{3}\right), 25.87\left(2 \mathrm{Si}-\mathrm{C}\left(\mathrm{CH}_{3}\right)_{3}\right), 25.12\left(\mathrm{~d}, J_{1}{ }^{\prime}-\mathrm{P}=141.8 \mathrm{~Hz}, \mathrm{C}-1^{\prime}\right), 18.35\left(\mathrm{Si}-\mathrm{C}\left(\mathrm{CH}_{3}\right)_{3}\right)$, $18.17\left(\mathrm{Si}-\mathrm{C}\left(\mathrm{CH}_{3}\right)_{3}\right), 18.07\left(\mathrm{Si}-\mathrm{C}\left(\mathrm{CH}_{3}\right)_{3}\right),-4.41$ ( $\left.\mathrm{Si}-\mathrm{Me}\right),-4.59$ (2Si-Me), -5.39 (2Si-Me), -5.50 $(\mathrm{Si}-\mathrm{Me}) ;{ }^{31} \mathrm{P}-\mathrm{NMR}\left(101 \mathrm{MHz}, \mathrm{CDCl}_{3}\right) \delta$ 32.05; MS $\left(\mathrm{CI}_{-N H}\right) \mathrm{m} / \mathrm{z} 713(100 \%)[\mathrm{M}+\mathrm{H}]^{+}, 730$ $(45 \%)\left[\mathrm{M}+\mathrm{NH}_{4}\right]^{+}$; El. Anal. for $\mathrm{C}_{32} \mathrm{H}_{69} \mathrm{O}_{9} \mathrm{PSi}_{3}$ : calc. (\%) C 53.90 H 9.75. mes. C 53.73 H 9.94.

\section{Dimethyl (2,5,6-tri-O-tert-butyldimethylsilyl-1,3-dienyl-D-galactofuranosyl)methane} phosphonate (8). ${ }^{1} \mathbf{H}-\mathrm{NMR}\left(400 \mathrm{MHz}, \mathrm{CDCl}_{3}\right) \delta 5.99$ (s, 1H, H-3), 4.60 (dd, $J_{5-6 \mathrm{a}}=5.6 \mathrm{~Hz}, J_{5-6 \mathrm{~b}}$ $=6.7 \mathrm{~Hz}, 1 \mathrm{H}, \mathrm{H}-5), 3.82\left(\mathrm{ABX}, J_{5-6 \mathrm{a}}=5.6 \mathrm{~Hz}, J_{6 \mathrm{a}-6 \mathrm{~b}}=10.0 \mathrm{~Hz}, 1 \mathrm{H}, \mathrm{H}-6 \mathrm{a}\right), 3.74\left(2 \mathrm{~d}, J_{\mathrm{H}-\mathrm{P}}=11.0\right.$ et $10.8 \mathrm{~Hz}, 6 \mathrm{H}, \mathrm{OMe}), 3.71\left(\mathrm{ABX}, J_{5-6 \mathrm{~b}}=6.7 \mathrm{~Hz}, J_{6 \mathrm{a}-6 \mathrm{~b}}=10.0 \mathrm{~Hz}, 1 \mathrm{H}, \mathrm{H}-6 \mathrm{~b}\right), 3.18\left(\mathrm{AX}, J_{1}{ }^{\prime}-\mathrm{P}=\right.$ 19.9 Hz, 2H, H-1'a, H-1'b), 0.99 (s, 9H, Si-tBu), 0.89 (s, 9H, Si-tBu), 0.88 (s, 9H, Si-tBu), 0.19 (s, 3H, Si-Me), 0.18 (s, 3H, Si-Me), 0.09 (s, 3H, Si-Me), 0.05 (s, 3H, Si-Me), 0.04 (s, 3H, Si-Me), 0.02 (s, 3H, Si-Me); ${ }^{13} \mathrm{C}-\mathrm{NMR}\left(100 \mathrm{MHz}, \mathrm{CDCl}_{3}\right) \delta 152.50\left(\mathrm{~d}, J_{4-\mathrm{P}}=4.3 \mathrm{~Hz}, \mathrm{C}-4\right), 140.06(\mathrm{~d}$, $J_{1,2-\mathrm{P}}=10.1 \mathrm{~Hz}, \mathrm{C}-1$ and C-2), $129.46\left(\mathrm{~d}, J_{1,2-\mathrm{P}}=14.2 \mathrm{~Hz}, \mathrm{C}-1\right.$ and C-2), $75.62\left(\mathrm{~d}, J_{3-\mathrm{P}}=3.8 \mathrm{~Hz}\right.$, 
C-3), $70.30\left(\mathrm{~d}, J_{5-\mathrm{P}}=1.3 \mathrm{~Hz}, \mathrm{C}-5\right), 66.79\left(\mathrm{~d}, J_{6-\mathrm{P}}=1.4 \mathrm{~Hz}, \mathrm{C}-6\right), 52.65$ (d, $\left.J_{\mathrm{C}-\mathrm{P}}=6.5 \mathrm{~Hz}, \mathrm{OMe}\right)$, $52.62\left(\mathrm{~d}, J_{\mathrm{C}-\mathrm{P}}=6.5 \mathrm{~Hz}, \mathrm{OMe}\right), 25.85\left(\mathrm{Si}-\mathrm{C}\left(\mathrm{CH}_{3}\right)_{3}\right), 25.71\left(\mathrm{Si}-\mathrm{C}\left(\mathrm{CH}_{3}\right)_{3}\right), 25.56\left(\mathrm{Si}-\mathrm{C}\left(\mathrm{CH}_{3}\right)_{3}\right)$, $22.60\left(\mathrm{~d}, J_{1}{ }^{\prime}-\mathrm{P}=143.8 \mathrm{~Hz}, \mathrm{C}-1^{\prime}\right), 18.27\left(\mathrm{Si}-\mathrm{C}\left(\mathrm{CH}_{3}\right)_{3}\right), 18.20\left(\mathrm{Si}-\mathrm{C}\left(\mathrm{CH}_{3}\right)_{3}\right), 17.97\left(\mathrm{Si}-\mathrm{C}\left(\mathrm{CH}_{3}\right)_{3}\right)$, 4.67 (Si-Me), -4.69 (Si-Me), -4.93 (Si-Me), -5.05 (Si-Me), -5.43 (Si-Me), -5.51 (Si-Me); ${ }^{31} \mathrm{P}-$ NMR (101 MHz, CDCl3) $\delta$ 25.84; MS $\left(\mathrm{CI}_{-N H}\right) \mathrm{m} / \mathrm{z} 609(10 \%)[\mathrm{M}+\mathrm{H}]^{+}, 626(90 \%)[\mathrm{M}+$ $\left.\mathrm{NH}_{4}\right]^{+}$; HRMS for $\mathrm{C}_{27} \mathrm{H}_{61} \mathrm{O}_{7} \mathrm{NPSi}_{3}$ : calc. 626.3494 mes. 626.3492 .

(1(1')Z)- 2-O-tert-butyldimethylsilyl-1- ～(dimethoxyphosphoryl)methylidene-5,6-O(dimethyl-methylidene)- D-galactofuranose (9). To a solution of exoglycal 7 (269 $\mathrm{mg}, 0.52$ $\mathrm{mmol})$ in anhydrous $\mathrm{MeOH}(10 \mathrm{~mL})$ was added at $0^{\circ} \mathrm{C} \mathrm{NaH}(62 \mathrm{mg}, 60 \%$ in mineral oil $)$. The solution was stirred 12 hours at room temperature followed by addition of silica gel ( $3 \mathrm{~g})$. The solution was filtered, concentrated and purified by chromatography on silica gel (AcOEt) to yield $9(151 \mathrm{mg}, 67 \%)$ as a white solid. $[\alpha]_{\mathrm{D}}{ }^{20}-2.9\left(\mathrm{c} 1.3, \mathrm{CHCl}_{3}\right)$; m.p. $128-129^{\circ} \mathrm{C} ;{ }^{1} \mathrm{H}-\mathrm{NMR}(400$ $\left.\mathrm{MHz}, \mathrm{CDCl}_{3}\right) \delta 4.78\left(\mathrm{~d}, J_{3-\mathrm{OH}}=9.3 \mathrm{~Hz}, 1 \mathrm{H}, \mathrm{OH}-3\right), 4.69\left(\mathrm{ddd}, J_{1}{ }^{\prime}-2=1.6 \mathrm{~Hz}, J_{2-3}=8.3 \mathrm{~Hz}, J_{2-\mathrm{P}}=\right.$ $4.4 \mathrm{~Hz}, 1 \mathrm{H}, \mathrm{H}-2), 4.59$ (dd, $J_{1^{\prime}-2}=1.6 \mathrm{~Hz}, J_{1}{ }^{\prime}-\mathrm{P}=11.3 \mathrm{~Hz}, 1 \mathrm{H}, \mathrm{H}-1^{\prime}$ '), 4.37-4.30 (m, 2H, H-4, H5), $4.10\left(\mathrm{ABX}, J_{5-6 \mathrm{a}}=6.4 \mathrm{~Hz}, J_{6 \mathrm{a}-6 \mathrm{~b}}=8.4 \mathrm{~Hz}, 1 \mathrm{H}, \mathrm{H}-6 \mathrm{a}\right), 4.00\left(\mathrm{dd}, J_{2-3}=8.3 \mathrm{~Hz}, 1 \mathrm{H}, \mathrm{H}-3\right), 3.99$ $\left(\mathrm{ABX}, J_{5-6 \mathrm{~b}}=7.8 \mathrm{~Hz}, J_{6 \mathrm{a}-6 \mathrm{~b}}=8.4 \mathrm{~Hz}, 1 \mathrm{H}, \mathrm{H}-6 \mathrm{~b}\right), 3.74\left(\mathrm{~d}, J_{\mathrm{H}-\mathrm{P}}=11.4 \mathrm{~Hz}, 3 \mathrm{H}, \mathrm{OMe}\right), 3.71\left(\mathrm{~d}, J_{\mathrm{H}-\mathrm{P}}\right.$ $=11.2 \mathrm{~Hz}, 3 \mathrm{H}, \mathrm{OMe}), 1.46,1.38\left(2 \mathrm{~s}, 6 \mathrm{H}, \mathrm{C}\left(\mathrm{CH}_{3}\right)_{2}\right.$, acetonide), $0.96(\mathrm{~s}, 9 \mathrm{H}, \mathrm{Si}-\mathrm{Bu}), 0.23(\mathrm{~s}, 3 \mathrm{H}$, Si-Me), 0.17 (s, 3H, Si-Me); ${ }^{13} \mathrm{C}-\mathrm{NMR}\left(100 \mathrm{MHz}, \mathrm{CDCl}_{3}\right) \delta 173.51$ (d, $\left.J_{1-\mathrm{P}}=1.2 \mathrm{~Hz}, \mathrm{C}-1\right)$, $109.60\left(\mathrm{C}\left(\mathrm{CH}_{3}\right)_{2}\right.$, acetonide), $85.55(\mathrm{C}-4), 80.15\left(\mathrm{~d}, J_{1}{ }^{\prime}-\mathrm{P}=197.9 \mathrm{~Hz}, \mathrm{C}-1\right.$ ' $), 77.94\left(\mathrm{~d}, J_{2-\mathrm{P}}=12.5\right.$ $\mathrm{Hz}, \mathrm{C}-2), 75.98$ (C-3), 74.62 (C-5), 65.00 (C-6), 53.03 (d, $\left.J_{\mathrm{C}-\mathrm{P}}=5.8 \mathrm{~Hz}, \mathrm{OMe}\right), 51.60$ (d, $J_{\mathrm{C}-\mathrm{P}}=$

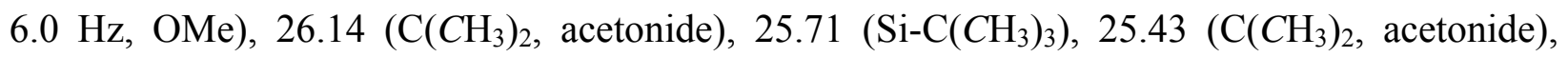
$17.93\left(\mathrm{Si}-\mathrm{C}\left(\mathrm{CH}_{3}\right)_{3}\right),-4.23$ (Si-Me), -5.06 (Si-Me); ${ }^{31} \mathrm{P}-\mathrm{NMR}\left(101 \mathrm{MHz}, \mathrm{CDCl}_{3}\right) \delta 21.02$; MS $\left(\mathrm{CI}-\mathrm{NH}_{3}\right) \mathrm{m} / \mathrm{z} 438(100 \%)[\mathrm{M}+\mathrm{H}]^{+}, 456(10 \%)\left[\mathrm{M}+\mathrm{NH}_{4}\right]^{+}$; El. Anal. for $\mathrm{C}_{18} \mathrm{H}_{35} \mathrm{O}_{8} \mathrm{PSi}$ : calc. (\%) C 49.30 H 8.04. mes. C 49.29 H 8.08.

Dimethyl(2-O-tert-butyldimethylsilyl-5,6-O-(dimethyl-methylidene)- $\alpha$-D-galactofuran -osyl) methanephosphonate (9a). The "general procedure for exoglycals hydrogenation" was applied. After chromatography on silica gel (Cy/AcOEt : 2/1), compound 9a (89\%) was isolated as a colourless oil. $[\alpha]_{\mathrm{D}}{ }^{21}-6.1\left(\mathrm{c} 0.76, \mathrm{CHCl}_{3}\right) ;{ }^{1} \mathrm{H}-\mathrm{NMR}\left(400 \mathrm{MHz}, \mathrm{CDCl}_{3}\right) \delta 4.37$ (tdd, $J_{1-2}=3.4 \mathrm{~Hz}$, $J_{1}{ }^{\prime}$, b-1 $\left.=6.5 \mathrm{~Hz}, J_{1-\mathrm{P}}=9.3 \mathrm{~Hz}, 1 \mathrm{H}, \mathrm{H}-1\right), 4.30\left(\mathrm{q}, J_{4-5}=J_{5-6 \mathrm{a}}=J_{5-6 \mathrm{~b}}=6.7 \mathrm{~Hz}, 1 \mathrm{H}, \mathrm{H}-5\right), 4.06\left(\mathrm{~d}, J_{1-}\right.$ $\left.{ }_{2}=3.4 \mathrm{~Hz}, 1 \mathrm{H}, \mathrm{H}-2\right), 4.03\left(\mathrm{ABX}, J_{5-6 \mathrm{a}}=6.9 \mathrm{~Hz}, J_{6 \mathrm{a}-6 \mathrm{~b}}=8.4 \mathrm{~Hz}, 1 \mathrm{H}, \mathrm{H}-6 \mathrm{a}\right), 3.92\left(\mathrm{ABX}, J_{5-6 \mathrm{~b}}=\right.$ $\left.6.4 \mathrm{~Hz}, J_{6 \mathrm{a}-6 \mathrm{~b}}=8.4 \mathrm{~Hz}, 1 \mathrm{H}, \mathrm{H}-6 \mathrm{~b}\right), 3.88\left(\mathrm{~d}, J_{3-4}=3.3 \mathrm{~Hz}, 1 \mathrm{H}, \mathrm{H}-3\right), 3.81\left(\mathrm{dd}, J_{3-4}=3.3 \mathrm{~Hz}, J_{4-5}=\right.$ $6.9 \mathrm{~Hz}, 1 \mathrm{H}, \mathrm{H}-4), 3.78$ (d, J $\left.J_{\mathrm{H}-\mathrm{P}}=10.9 \mathrm{~Hz}, 3 \mathrm{H}, \mathrm{OMe}\right), 3.75$ (d, $\left.J_{\mathrm{H}-\mathrm{P}}=10.9 \mathrm{~Hz}, 3 \mathrm{H}, \mathrm{OMe}\right), 2.21$

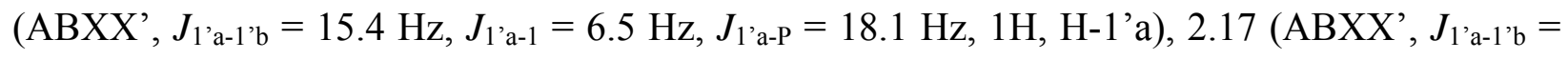
$\left.15.4 \mathrm{~Hz}, J_{1}{ }^{\prime} \mathrm{b}-1=6.5 \mathrm{~Hz}, J_{1}{ }^{\prime}-\mathrm{P}=20.9 \mathrm{~Hz}, 1 \mathrm{H}, \mathrm{H}-1{ }^{\prime} \mathrm{b}\right), 1.46,1.37$ (2s, 6H, C(CH$)_{2}$, acetonide), 0.93 (s, 9H, Si-tBu), 0.16 (s, 3H, Si-Me), 0.14 (s, 3H, Si-Me); ${ }^{13} \mathrm{C}-\mathrm{NMR}\left(100 \mathrm{MHz}, \mathrm{CDCl}_{3}\right) \delta$ $109.63\left(\mathrm{C}\left(\mathrm{CH}_{3}\right)_{2}\right.$, acetonide), $86.62(\mathrm{C}-4), 79.61(\mathrm{C}-3), 79.19\left(\mathrm{~d}, J_{2-\mathrm{P}}=8.5 \mathrm{~Hz}, \mathrm{C}-2\right), 76.11(\mathrm{C}-1)$, 75.90 (C-5), $65.44(\mathrm{C}-6), 52.78\left(\mathrm{~d}, J_{\mathrm{C}-\mathrm{P}}=6.4 \mathrm{~Hz}, \mathrm{OMe}\right), 52.03\left(\mathrm{~d}, J_{\mathrm{C}-\mathrm{P}}=6.4 \mathrm{~Hz}, \mathrm{OMe}\right), 26.57$ $\left(\mathrm{C}\left(\mathrm{CH}_{3}\right)_{2}\right.$, acetonide), $25.73\left(\mathrm{Si}-\mathrm{C}\left(\mathrm{CH}_{3}\right)_{3}\right), 24.85\left(\mathrm{~d}, J_{1}{ }^{\prime} \mathrm{P}=141.4 \mathrm{~Hz}, \mathrm{C}-1^{\prime}\right), 24.14\left(\mathrm{C}^{\prime} \mathrm{CH}_{3}\right)_{2}$, acetonide), $18.00\left(\mathrm{Si}-\mathrm{C}\left(\mathrm{CH}_{3}\right)_{3}\right),-4.50(\mathrm{Si}-\mathrm{Me}),-5.19(\mathrm{Si}-\mathrm{Me}) ;{ }^{31} \mathrm{P}-\mathrm{NMR}\left(101 \mathrm{MHz}, \mathrm{CDCl}_{3}\right) \delta$ 
32.27; $\mathrm{MS}\left(\mathrm{CI}_{\left.-\mathrm{NH}_{3}\right)}\right) \mathrm{m} / \mathrm{z} 441(100 \%)[\mathrm{M}+\mathrm{H}]^{+}, 458(75 \%)\left[\mathrm{M}+\mathrm{NH}_{4}\right]^{+}$; HRMS for $\mathrm{C}_{18} \mathrm{H}_{38} \mathrm{O}_{8} \mathrm{PSi}$ : calc. 441.2074 mes. 441.2065.

(10) (1(1')Z)-1-(dimethoxyphosphoryl)methylidene-5,6-O-(dimethyl-methylidene)-3-Opivaloyl-D-galactofuranose. To a solution of $7(1.28 \mathrm{~g}, 2.45 \mathrm{mmol})$ in distilled THF (10 $\mathrm{mL})$ was added tetrabutyl ammonium trihydrate $(850 \mathrm{mg}, 2.69 \mathrm{mmol})$ at $0^{\circ} \mathrm{C}$. The solution was stirred 30 minutes at $0^{\circ} \mathrm{C}$ and concentrated. Compound $10(727 \mathrm{mg}, 73 \%)$ was obtained as a white solid after chromatography on silica gel (AcOEt). $[\alpha]_{\mathrm{D}}{ }^{19}+78.8$ (c $\left.0.9, \mathrm{CHCl}_{3}\right)$; m.p. $77-78^{\circ} \mathrm{C} ;{ }^{1} \mathrm{H}-\mathrm{NMR}$ $\left(400 \mathrm{MHz}, \mathrm{CDCl}_{3}\right) \delta 5.01$ (d, 1H, H-3), 4.76 (d, $\left.J_{1}{ }^{\prime}-\mathrm{P}=9.9 \mathrm{~Hz}, 1 \mathrm{H}, \mathrm{H}-1^{\prime}\right), 4.71(\mathrm{~s}, 1 \mathrm{H}, \mathrm{OH}-2)$, $4.49(\mathrm{~m}, 1 \mathrm{H}, \mathrm{H}-2), 4.41-4.37(\mathrm{~m}, 2 \mathrm{H}, \mathrm{H}-4, \mathrm{H}-5), 4.08\left(\mathrm{ABX}, J_{5-6 \mathrm{a}}=6.5 \mathrm{~Hz}, J_{6 \mathrm{a}-6 \mathrm{~b}}=8.3 \mathrm{~Hz}, 1 \mathrm{H}\right.$, $\mathrm{H}-6 \mathrm{a}), 4.01\left(\mathrm{ABX}, J_{5-6 \mathrm{~b}}=7.7 \mathrm{~Hz}, J_{6 \mathrm{a}-6 \mathrm{~b}}=8.3 \mathrm{~Hz}, 1 \mathrm{H}, \mathrm{H}-6 \mathrm{~b}\right), 3.68\left(2 \mathrm{~d}, J_{\mathrm{H}-\mathrm{P}}=11.3 \mathrm{~Hz}, 6 \mathrm{H}, \mathrm{OMe}\right)$, 1.39, $1.34\left(2 \mathrm{~s}, 6 \mathrm{H}, \mathrm{C}\left(\mathrm{CH}_{3}\right)_{2}\right.$, acetonide), 1.15 (s, 9H, Piv); ${ }^{13} \mathrm{C}-\mathrm{NMR}\left(100 \mathrm{MHz}, \mathrm{CDCl}_{3}\right) \delta 177.88$ $(\mathrm{C}=\mathrm{O}, \mathrm{Piv}), 172.33\left(\mathrm{~d}, J_{1-\mathrm{P}}=3.1 \mathrm{~Hz}, \mathrm{C}-1\right), 110.48\left(\mathrm{C}\left(\mathrm{CH}_{3}\right)_{2}\right.$, acetonide), $85.40(\mathrm{C}-4), 82.04$ (d, $J_{1}{ }^{\prime}-\mathrm{P}=195.5 \mathrm{~Hz}, \mathrm{C}-1^{\prime}$ ) $, 78.55\left(\mathrm{~d}, J_{3-\mathrm{P}}=1.3 \mathrm{~Hz}, \mathrm{C}-3\right), 76.19$ (d, $\left.J_{2-\mathrm{P}}=14.3 \mathrm{~Hz}, \mathrm{C}-2\right), 74.96(\mathrm{C}-5)$, $65.05(\mathrm{C}-6), 52.17\left(\mathrm{~d}, J_{\mathrm{C}-\mathrm{P}}=5.5 \mathrm{~Hz}, \mathrm{OMe}\right), 52.02\left(\mathrm{~d}, J_{\mathrm{C}-\mathrm{P}}=5.8 \mathrm{~Hz}, \mathrm{OMe}\right), 38.47\left(\mathrm{CO}-\mathrm{C}\left(\mathrm{CH}_{3}\right)_{3}\right.$, Piv), $26.75\left(\mathrm{CO}-\mathrm{C}\left(\mathrm{CH}_{3}\right)_{3}, \mathrm{Piv}\right), 25.40,25.29\left(\mathrm{C}\left(\mathrm{CH}_{3}\right)_{2}\right.$, acetonide); ${ }^{31} \mathrm{P}-\mathrm{NMR}\left(101 \mathrm{MHz}, \mathrm{CDCl}_{3}\right)$ $\delta$ 20.36; MS $\left(\mathrm{CI}_{\left.-\mathrm{NH}_{3}\right)}\right) \mathrm{m} / \mathrm{z} 409(100 \%)[\mathrm{M}+\mathrm{H}]^{+}, 426(30 \%)\left[\mathrm{M}+\mathrm{NH}_{4}\right]^{+}$; El. Anal. for $\mathrm{C}_{17} \mathrm{H}_{29} \mathrm{O}_{9} \mathrm{P}$ : calc. (\%) C $50.00 \mathrm{H} 7.16$. mes. C $49.87 \mathrm{H} 7.22$.

Dimethyl (5,6-O-(dimethyl-methylidene)-3-O-pivaloyl- $\alpha$-D-galactofuranosyl)methane phosphonate (10a). The "general procedure for exoglycals hydrogenation" was applied. After chromatography on silica gel (Cy/AcOEt: 2/1), compound 10a (56\%) was isolated as a colourless oil $(\alpha / \beta: 80 / 20)$. ${ }^{1} \mathrm{H}-\mathrm{NMR}\left(400 \mathrm{MHz}, \mathrm{CDCl}_{3}\right) \delta 5.08\left(\mathrm{t}, J_{2-3}=J_{3-4}=3.0 \mathrm{~Hz}, 0.25 \mathrm{H}, \mathrm{H}-\right.$ $\left.3_{\beta}\right), 5.00\left(\mathrm{~s}, 1 \mathrm{H}, \mathrm{H}-3_{\alpha}\right), 4.46\left(\mathrm{td}, J_{4-5}=2.0 \mathrm{~Hz}, J_{5-6 \mathrm{a}, \mathrm{b}}=8.2 \mathrm{~Hz}, 1 \mathrm{H}, \mathrm{H}-5_{\alpha}\right), 4.42\left(\mathrm{dd}, J_{5-6 \mathrm{a}}=3.1 \mathrm{~Hz}\right.$, $\left.J_{5-6 \mathrm{a}}=7.1 \mathrm{~Hz}, 0.25 \mathrm{H}, \mathrm{H}-5_{\beta}\right), 4.36\left(\mathrm{tdd}, J_{1-2}=1.9 \mathrm{~Hz}, J_{1}\right.$ 'a,b-1 $\left.=6.3 \mathrm{~Hz}, J_{1-\mathrm{P}}=10.6 \mathrm{~Hz}, 0.25 \mathrm{H}, \mathrm{H}-1_{\beta}\right)$, $4.30\left(\mathrm{tdd}, J_{1}{ }^{\prime} \mathrm{b}-1=2.6 \mathrm{~Hz}, J_{1-2}=J_{1}{ }^{\prime} \mathrm{a}-1=6.8 \mathrm{~Hz}, J_{1-\mathrm{P}}=13.9 \mathrm{~Hz}, 1 \mathrm{H}, \mathrm{H}-1_{\alpha}\right), 4.15\left(\mathrm{~d}, J_{2-3}=3.0 \mathrm{~Hz}\right.$, $\left.0.25 \mathrm{H}, \mathrm{H}-2_{\beta}\right), 4.00\left(\mathrm{~m}, 1.25, \mathrm{H}-6 \mathrm{a}_{\alpha}-6 \mathrm{a}_{\beta}\right), 3.98$ (d, $\left.J_{1-2}=6.8 \mathrm{~Hz}, 1 \mathrm{H}, \mathrm{H}-2_{\alpha}\right), 3.99$ (m, 1.5H, H$\left.6 \mathrm{~b}_{. \alpha} \mathrm{H}-6 \mathrm{~b}_{\beta} \mathrm{H}-4_{\beta}\right), 3.82\left(\mathrm{~d}, J_{4-5}=2.0 \mathrm{~Hz}, 1 \mathrm{H}, \mathrm{H}-4_{\alpha}\right), 3.80,3.78\left(2 \mathrm{~d}, J_{\mathrm{H}-\mathrm{P}}=11.1,11.0 \mathrm{~Hz}, 6 \mathrm{H}, \mathrm{OMe}_{\alpha}\right)$, $3.78,3.74\left(2 \mathrm{~d}, J_{\mathrm{H}-\mathrm{P}}=11.0,10.9 \mathrm{~Hz}, 0.75 \mathrm{H}, \mathrm{OMe}_{\beta}\right), 2.26\left(\mathrm{ABXX}^{\prime}, J_{1}{ }^{\prime}\right.$ a- $1=6.8 \mathrm{~Hz}, J_{1}$ 'a-P $=18.4 \mathrm{~Hz}$, 2.75H, H-1' $\mathrm{a}_{\alpha \beta} \mathrm{H}-1$ ' $\mathrm{b}_{\alpha \beta}, 1.45,1.41\left(2 \mathrm{~s}, 6 \mathrm{H}, \mathrm{C}\left(\mathrm{CH}_{3}\right)_{2}\right.$, acetonide $\left.{ }_{\alpha}\right), 1.46,1.40\left(2 \mathrm{~s}, 1.5 \mathrm{H}, \mathrm{C}\left(\mathrm{CH}_{3}\right)_{2}\right.$, acetonide $\left.{ }_{\alpha}\right), 1.23\left(\mathrm{~s}, 2.25 \mathrm{H}, \mathrm{Piv}_{\beta}\right), 1.21\left(\mathrm{~s}, 9 \mathrm{H}, \mathrm{Piv}_{\alpha}\right) ;{ }^{13} \mathrm{C}-\mathrm{NMR}\left(100 \mathrm{MHz}, \mathrm{CDCl}_{3}\right) \delta 177.13$

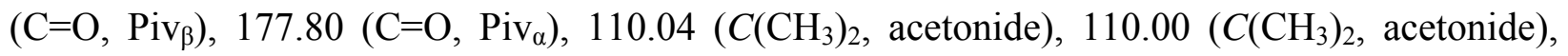
$82.23\left(\mathrm{C}-4_{\alpha}\right), 81.97\left(\mathrm{C}-4_{\beta}\right), 81.79\left(\mathrm{C}-3_{\beta}\right), 80.94\left(\mathrm{C}-3_{\alpha}\right), 80.71\left(\mathrm{C}-1_{\beta}\right), 79.45\left(\mathrm{~d}, J_{2-\mathrm{P}}=6.6 \mathrm{~Hz}, \mathrm{C}-\right.$ $\left.2_{\beta}\right), 77.37\left(\mathrm{C}-1_{\alpha}\right), 76.08\left(\mathrm{C}-5_{\beta}\right), 75.68\left(\mathrm{C}-5_{\alpha}\right), 74.83\left(\mathrm{~d}, J_{2-\mathrm{P}}=8.5 \mathrm{~Hz}, \mathrm{C}-2_{\alpha}\right), 65.56\left(\mathrm{C}-6_{\beta}\right), 65.51$ $\left(\mathrm{C}-6_{\alpha}\right), 52.63\left(\mathrm{~d}, J_{\mathrm{C}-\mathrm{P}}=6.1 \mathrm{~Hz}, \mathrm{OMe}_{\alpha}\right), 52.61\left(\mathrm{~d}, J_{\mathrm{C}-\mathrm{P}}=6.6 \mathrm{~Hz}, \mathrm{OMe}_{\beta}\right), 52.51\left(\mathrm{~d}, J_{\mathrm{C}-\mathrm{P}}=6.3 \mathrm{~Hz}\right.$, $\left.\mathrm{OMe}_{\beta}\right), 52.24\left(\mathrm{~d}, J_{\mathrm{C}-\mathrm{P}}=6.4 \mathrm{~Hz}, \mathrm{OMe}_{\alpha}\right), 38.48\left(\mathrm{CO}-\mathrm{C}\left(\mathrm{CH}_{3}\right)_{3}, \mathrm{Piv}_{\alpha \beta}, 29.63\left(\mathrm{~d}, J_{1}{ }^{\prime}-\mathrm{P}=137.9 \mathrm{~Hz}, \mathrm{C}-\right.\right.$ $\left.1_{\beta}^{\prime}\right), 26.96\left(\mathrm{CO}-\mathrm{C}\left(\mathrm{CH}_{3}\right)_{3}, \mathrm{Piv}_{\beta}\right), 26.92\left(\mathrm{CO}-\mathrm{C}\left(\mathrm{CH}_{3}\right)_{3}, \mathrm{Piv}_{\alpha}\right), 25.85,25.18\left(\mathrm{C}_{\left(\mathrm{CH}_{3}\right)_{2} \text {, acetonide }}\right)$, 25.70, $25.60\left(\mathrm{C}_{\left(\mathrm{CH}_{3}\right)_{2} \text {, acetonide }}\right), 24.48\left(\mathrm{~d}, J_{1}\right.$ '-P $\left.=141.0 \mathrm{~Hz}, \mathrm{C}-1{ }^{\prime}{ }_{\alpha}\right) ;{ }^{31} \mathrm{P}-\mathrm{NMR}(101 \mathrm{MHz}$, $\left.\mathrm{CDCl}_{3}\right) \delta 31.27(\alpha), 30.20(\beta) ; \mathrm{MS}\left(\mathrm{CI}-\mathrm{NH}_{3}\right) \mathrm{m} / \mathrm{z} 379(100 \%)[\mathrm{M}-32+\mathrm{H}]^{+}, 411(27 \%)[\mathrm{M}+$ $\mathrm{H}]^{+}$; HRMS for $\mathrm{C}_{16} \mathrm{H}_{28} \mathrm{O}_{8} \mathrm{P}$ : calc. 379.1522 mes. 379.1517 .

(1(1')Z)-2-O-formyl-1-(dimethoxyphosphoryl)methylidene-5,6-O-(dimethyl-methyl -idene)3-O-pivaloyl-D-galactofuranose (11). To a solution of DCC (378 mg, $1.83 \mathrm{mmol})$ in anhydrous 
$\mathrm{CH}_{2} \mathrm{Cl}_{2}(15 \mathrm{~mL})$ was added, at $0^{\circ} \mathrm{C}$ under argon, formic acid $(65 \mu \mathrm{L}, 1.73 \mathrm{mmol})$. After 10 minutes at $0^{\circ} \mathrm{C}$, a solution of $\mathbf{1 0}$ in anhydrous $\mathrm{CH}_{2} \mathrm{Cl}_{2}(3 \mathrm{~mL})$ was added. The resulting mixture was stirred overnight at room temperature, concentrated, dried 1 hour under vacuum, dissolved in $\mathrm{Et}_{2} \mathrm{O}$ and filtered on filter paper. The filtrate was concentrated under rotary evaporation and the resulting crude residue was purified by chromatography on silica gel with AcOEt as eluent, to yield $11(118 \mathrm{mg}, 79 \%)$ as a colourless oil. $[\alpha]_{\mathrm{D}}{ }^{21}+67.9\left(\mathrm{c} 1.2, \mathrm{CHCl}_{3}\right) ;{ }^{1} \mathrm{H}-\mathrm{NMR}(400 \mathrm{MHz}$, $\left.\mathrm{CDCl}_{3}\right) \delta 8.14(\mathrm{~s}, 1 \mathrm{H}, \mathrm{CHO}), 5.94\left(\mathrm{tdd}, J_{1^{\prime}-2}=1.4 \mathrm{~Hz}, J_{2-3}=J_{2-\mathrm{P}}=4.1 \mathrm{~Hz}, J_{2-\mathrm{H}(\mathrm{OCHO})}=1.2 \mathrm{~Hz}\right.$, $1 \mathrm{H}, \mathrm{H}-2), 5.35$ (t, $\left.J_{2-3}=J_{3-4}=4.1 \mathrm{~Hz}, 1 \mathrm{H}, \mathrm{H}-3\right), 4.72\left(\mathrm{dd}, J_{1^{\prime}-2}=1.4 \mathrm{~Hz}, J_{1^{\prime}-\mathrm{P}}=8.2 \mathrm{~Hz}, 1 \mathrm{H}, \mathrm{H}-1^{\prime}\right.$ ), 4.47-4.39 (m, 2H, H-4, H-5), $4.11\left(\mathrm{ABX}, J_{5-6 \mathrm{a}}=6.6 \mathrm{~Hz}, J_{6 \mathrm{a}-6 \mathrm{~b}}=8.5 \mathrm{~Hz}, 1 \mathrm{H}, \mathrm{H}-6 \mathrm{a}\right), 4.03(\mathrm{ABX}$, $\left.J_{5-6 \mathrm{~b}}=6.4 \mathrm{~Hz}, J_{6 \mathrm{a}-6 \mathrm{~b}}=8.5 \mathrm{~Hz}, 1 \mathrm{H}, \mathrm{H}-6 \mathrm{~b}\right), 3.78\left(\mathrm{~d}, J_{\mathrm{H}-\mathrm{P}}=11.4 \mathrm{~Hz}, 3 \mathrm{H}, \mathrm{OMe}\right), 3.76\left(\mathrm{~d}, J_{\mathrm{H}-\mathrm{P}}=11.3\right.$ $\mathrm{Hz}, 3 \mathrm{H}, \mathrm{OMe}), 1.44,1.38\left(2 \mathrm{~s}, 6 \mathrm{H}, \mathrm{C}\left(\mathrm{CH}_{3}\right)_{2}\right.$, acetonide), 1.23 (s, 9H, Piv); ${ }^{13} \mathrm{C}-\mathrm{NMR}(100 \mathrm{MHz}$, $\left.\mathrm{CDCl}_{3}\right) \delta 177.59(\mathrm{C}=\mathrm{O}, \mathrm{Piv}), 166.35$ (d, $\left.J_{1-\mathrm{P}}=1.4 \mathrm{~Hz}, \mathrm{C}-1\right), 159.25(\mathrm{C}=\mathrm{O}, \mathrm{CHO}), 110.24$ $\left(C\left(\mathrm{CH}_{3}\right)_{2}\right.$, acetonide), $85.53(\mathrm{C}-4), 85.39\left(\mathrm{~d}, J_{1}{ }^{\prime}-\mathrm{P}=196.1 \mathrm{~Hz}, \mathrm{C}-1^{\prime}\right), 75.76\left(\mathrm{~d}, J_{2-\mathrm{P}}=14.4 \mathrm{~Hz}, \mathrm{C}-\right.$ 2), $75.57\left(\mathrm{~d}, J_{3-\mathrm{P}}=1.0 \mathrm{~Hz}, \mathrm{C}-3\right), 74.40$ (C-5), 64.87 (C-6), 52.52 (d, $\left.J_{\mathrm{C}-\mathrm{P}}=5.8 \mathrm{~Hz}, \mathrm{OMe}\right), 52.21$

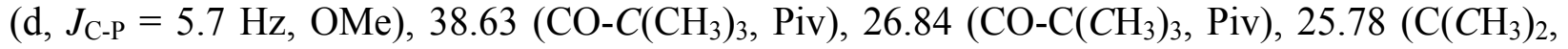

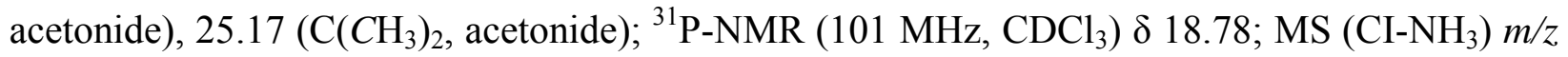
$437(100 \%)[\mathrm{M}+\mathrm{H}]^{+}, 454(20 \%)\left[\mathrm{M}+\mathrm{NH}_{4}\right]^{+}$; HRMS for $\mathrm{C}_{18} \mathrm{H}_{30} \mathrm{O}_{10} \mathrm{P}$ calc. 437.1577 mes. 437.1570 .

Dimethyl (2-O-formyl-5,6-O-(dimethyl-methylidene)-3-O-pivaloyl- $\alpha$-D-galactofuranosyl) methanephosphonate (11a). The "general procedure for exoglycals hydrogenation" was applied. After chromatography on silica gel (AcOEt), compound 11a (70\%) was isolated as a colourless oil $(\tilde{\alpha} / \beta: 82 / 18) .{ }^{1} \mathrm{H}-\mathrm{NMR}\left(400 \mathrm{MHz}, \mathrm{CDCl}_{3}\right) \delta 8.12\left(\mathrm{~s}, 1 \mathrm{H}, \mathrm{CHO}_{\alpha}\right), 8.07\left(\mathrm{~s}, 0.18 \mathrm{H}, \mathrm{CHO}_{\beta}\right), 5.30$ $\left(\mathrm{m}, 0.18 \mathrm{H}, \mathrm{H}-2_{\beta}\right), 5.28\left(\mathrm{~d}, J_{1-2}=3.4 \mathrm{~Hz}, J_{2-3}=0.7 \mathrm{~Hz}, 1 \mathrm{H}, \mathrm{H}-2_{\alpha}\right), 5.20\left(\mathrm{t}, J_{2-3}=J_{3-4}=3.4 \mathrm{~Hz}\right.$, $\left.0.18 \mathrm{H}, \mathrm{H}-3_{\beta}\right), 4.98\left(\mathrm{~d}, J_{3-4}=4.0 \mathrm{~Hz}, 1 \mathrm{H}, \mathrm{H}-3_{\alpha}\right), 4.53\left(\mathrm{~m}, 0.18 \mathrm{H}, \mathrm{H}-1_{\beta}\right), 4.41$ (ddd, $J_{1-2}=3.4 \mathrm{~Hz}$,

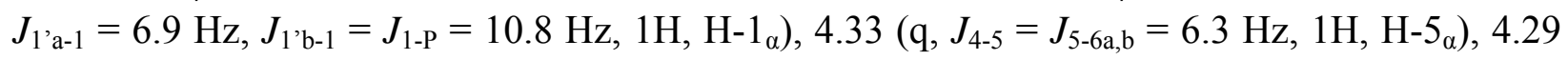
$\left(\mathrm{m}, 0.18 \mathrm{H}, \mathrm{H}-5_{\beta}\right), 4.05\left(\mathrm{ABX}, J_{5-6 \mathrm{a}}=6.3 \mathrm{~Hz}, J_{6 \mathrm{a}-6 \mathrm{~b}}=8.6 \mathrm{~Hz}, 1 \mathrm{H}, \mathrm{H}-6 \mathrm{a}_{\alpha}\right), 4.04\left(\mathrm{~m}, 0.18 \mathrm{H}, \mathrm{H}-6 \mathrm{a}_{\beta}\right)$, $3.97\left(\mathrm{~m}, 0.18 \mathrm{H}, \mathrm{H}-4_{\beta}\right), 3.87\left(\mathrm{~m}, 0.18 \mathrm{H}, \mathrm{H}-6 \mathrm{~b}_{\beta}\right), 3.86\left(\mathrm{ABX}, J_{5-6 \mathrm{~b}}=6.2 \mathrm{~Hz}, J_{6 \mathrm{a}-6 \mathrm{~b}}=8.6 \mathrm{~Hz}, 1 \mathrm{H}, \mathrm{H}-\right.$ $\left.6 \mathrm{~b}_{\alpha}\right), 3.80\left(\mathrm{dd}, J_{3-4}=4.0 \mathrm{~Hz}, J_{4-5}=6.2 \mathrm{~Hz}, 1 \mathrm{H}, \mathrm{H}-4_{\alpha}\right), 3.77,3.75\left(2 \mathrm{~d}, J_{\mathrm{H}-\mathrm{P}}=11.1,10.9 \mathrm{~Hz}, 7.1 \mathrm{H}\right.$, $\left.\mathrm{OMe}_{\alpha \beta}\right), 2.26-2.17\left(\mathrm{~m}, 0.36 \mathrm{H}, \mathrm{H}-1\right.$ ' $\left.\mathrm{a}_{\beta}, \mathrm{H}-1{ }^{\prime} \mathrm{b}_{\beta}\right), 2.26\left(\mathrm{ABXX}\right.$ ', $J_{1}{ }^{\prime} \mathrm{a}-1$ 'b $=15.3 \mathrm{~Hz}, J_{1}{ }^{\prime} \mathrm{a}-1=6.9 \mathrm{~Hz}$,

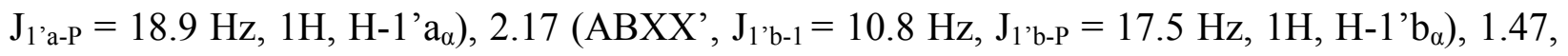
$1.38\left(2 \mathrm{~s}, 6 \mathrm{H}, \mathrm{C}\left(\mathrm{CH}_{3}\right)_{2}\right.$, acetonide $\left.{ }_{\beta}\right), 1.43,1.37\left(2 \mathrm{~s}, 1.08 \mathrm{H}, \mathrm{C}\left(\mathrm{CH}_{3}\right)_{2}\right.$, acetonide $\left.{ }_{\alpha}\right), 1.23(\mathrm{~s}, 9 \mathrm{H}$, $\left.\operatorname{Piv}_{\alpha}\right), 1.21\left(\mathrm{~s}, 1.62 \mathrm{H}, \mathrm{Piv}_{\beta}\right) ;{ }^{13} \mathrm{C}-\mathrm{NMR}\left(100 \mathrm{MHz}, \mathrm{CDCl}_{3}\right) \delta 177.10\left(\mathrm{C}=\mathrm{O}, \mathrm{Piv}_{\alpha \beta}\right), 159.53\left(\mathrm{CHO}_{\beta}\right)$, $159.33\left(\mathrm{CHO}_{\alpha}\right), 109.85\left(\mathrm{C}\left(\mathrm{CH}_{3}\right)_{2}\right.$, acetonide $\left.{ }_{\alpha \beta}\right), 84.11\left(\mathrm{C}-4_{\alpha}\right), 82.77\left(\mathrm{C}-4_{\beta}\right), 80.17\left(\mathrm{C}-2_{\beta}\right), 78.56$ $\left(\mathrm{C}-3_{\beta}\right), 78.33\left(\mathrm{C}-3_{\alpha}\right), 77.70\left(\mathrm{~d}, J_{1-\mathrm{P}}=2.9 \mathrm{~Hz}, \mathrm{C}-1_{\beta}\right), 77.16\left(\mathrm{~d}, J_{2-\mathrm{P}}=7.7 \mathrm{~Hz}, \mathrm{C}-2_{\alpha}\right), 75.69\left(\mathrm{C}-5_{\beta}\right)$, $75.20\left(\mathrm{C}-5_{\alpha}\right), 75.13\left(\mathrm{C}-1_{\alpha}\right), 65.50\left(\mathrm{C}-6_{\alpha}\right), 65.41\left(\mathrm{C}-6_{\beta}\right), 52.82\left(\mathrm{~d}, J_{\mathrm{C}-\mathrm{P}}=6.5 \mathrm{~Hz}, \mathrm{OMe}_{\alpha \beta}\right), 52.24(\mathrm{~d}$, $\left.J_{\mathrm{C}-\mathrm{P}}=6.5 \mathrm{~Hz}, \mathrm{OMe}_{\alpha \beta \beta}\right), 38.56\left(\mathrm{CO}-\mathrm{C}\left(\mathrm{CH}_{3}\right)_{3}, \mathrm{Piv}_{\alpha \cdot \beta}\right), 28.67\left(\mathrm{~d}, J_{1^{\prime}-\mathrm{P}}=140.7 \mathrm{~Hz}, \mathrm{C}-1^{\prime}{ }_{\beta}\right), 26.89(\mathrm{CO}-$ $\left.\mathrm{C}\left(\mathrm{CH}_{3}\right)_{3}, \mathrm{Piv}_{\alpha}\right), 26.86\left(\mathrm{CO}-\mathrm{C}\left(\mathrm{CH}_{3}\right)_{3}, \mathrm{Piv}_{\beta}\right), 26.21,25.48\left(\mathrm{C}_{\left(\mathrm{CH}_{3}\right)_{2} \text {, acetonide }}\right), 26.12,25.13$ $\left(\mathrm{C}_{\left(\mathrm{CH}_{3}\right)_{2} \text {, acetonide }}\right), 24.77\left(\mathrm{~d}, J_{1}{ }^{\prime}-\mathrm{P}=141.9 \mathrm{~Hz}, \mathrm{C}-1^{\prime}{ }_{\alpha}\right) ;{ }^{31} \mathrm{P}-\mathrm{NMR}\left(101 \mathrm{MHz}, \mathrm{CDCl}_{3}\right) \delta 29.37$ $(\alpha), 28.65(\beta) ; \mathrm{MS}\left(\mathrm{CI}-\mathrm{NH}_{3}\right) \mathrm{m} / \mathrm{z} 439[\mathrm{M}+\mathrm{H}]^{+}$; HRMS for $\mathrm{C}_{18} \mathrm{H}_{32} \mathrm{O}_{10} \mathrm{P}$ calc. 439.1733 mes. 439.1729. 
(1(1')Z)-2-O-Acetyl-1-(dimethoxyphosphoryl)methylidene-5,6-O-(dimethyl-methylidene)-3$\boldsymbol{O}$-pivaloyl-D-galactofuranose (12). To a solution of $\mathbf{1 0}(209 \mathrm{mg}, 0.51 \mathrm{mmol})$ in anhydrous pyridine $(10 \mathrm{~mL})$ was slowly added, at $0^{\circ} \mathrm{C}$ under argon, acetic anhydride $(145 \mu \mathrm{L}, 1.54 \mathrm{mmol})$. The resulting solution was stirred 12 hours at room temperature and the solvent was removed under vacuum. The residue was dissolved in AcOEt and washed with sat. $\mathrm{NaHCO}_{3}$ and water. The organic phases were dried over $\mathrm{MgSO}_{4}$, filtered and concentrated. Acetate $12(221 \mathrm{mg}$, $96 \%$ ) was obtained as a white solid after silica gel chromatography (AcOEt). $[\alpha]_{\mathrm{D}}{ }^{20}+31.7$ (c 0.8 , $\left.\mathrm{CHCl}_{3}\right)$; m.p. 81-82 ${ }^{\circ} \mathrm{C} ;{ }^{1} \mathrm{H}-\mathrm{NMR}\left(400 \mathrm{MHz}, \mathrm{CDCl}_{3}\right) \delta 5.83\left(\mathrm{td}, J_{1^{\prime}-2}=1.4 \mathrm{~Hz}, J_{2-\mathrm{P}}=J_{2-3}=4.0 \mathrm{~Hz}\right.$, $1 \mathrm{H}, \mathrm{H}-2), 5.30\left(\mathrm{t}, J_{2-3}=J_{3-4}=4.0 \mathrm{~Hz}, 1 \mathrm{H}, \mathrm{H}-3\right), 4.69\left(\mathrm{dd}, J_{1^{\prime}-2}=1.4 \mathrm{~Hz}, J_{1}{ }^{\prime}-\mathrm{P}=8.4 \mathrm{~Hz}, 1 \mathrm{H}, \mathrm{H}-1^{\prime}\right)$, 4.43-4.37 (m, 2H, H-4, H-5), $4.10\left(\mathrm{ABX}, J_{5-6 \mathrm{a}}=6.5 \mathrm{~Hz}, J_{6 \mathrm{a}-6 \mathrm{~b}}=8.4 \mathrm{~Hz}, 1 \mathrm{H}, \mathrm{H}-6 \mathrm{a}\right), 4.02(\mathrm{ABX}$, $\left.J_{5-6 \mathrm{~b}}=6.5 \mathrm{~Hz}, J_{6 \mathrm{a}-6 \mathrm{~b}}=8.4 \mathrm{~Hz}, 1 \mathrm{H}, \mathrm{H}-6 \mathrm{~b}\right), 3.77\left(\mathrm{~d}, J_{\mathrm{H}-\mathrm{P}}=11.3 \mathrm{~Hz}, 3 \mathrm{H}, \mathrm{OMe}\right), 3.76\left(\mathrm{~d}, J_{\mathrm{H}-\mathrm{P}}=11.3\right.$ $\mathrm{Hz}, 3 \mathrm{H}, \mathrm{OMe}), 2.15$ (s, 3H, Ac), 1.44, 1.37 (2s, 6H, $\mathrm{C}\left(\mathrm{CH}_{3}\right)_{2}$, acetonide), 1.22 (s, 9H, Piv); ${ }^{13} \mathrm{C}-$ NMR (100 MHz, $\left.\mathrm{CDCl}_{3}\right) \delta 177.58$ (C=O, Piv), 169.61 (C=O, Ac), 166.97 (d, $\left.J_{1-\mathrm{P}}=2.1 \mathrm{~Hz}, \mathrm{C}-1\right)$, $110.14\left(C\left(\mathrm{CH}_{3}\right)_{2}\right.$, acetonide), $85.19(\mathrm{C}-4), 84.71\left(\mathrm{~d}, J_{1}{ }^{\prime} \mathrm{P}=196.3 \mathrm{~Hz}, \mathrm{C}-1\right.$ ' $), 76.23$ (d, $J_{2-\mathrm{P}}=14.0$ $\mathrm{Hz}, \mathrm{C}-2), 75.51$ (d, $\left.J_{3-\mathrm{P}}=1.3 \mathrm{~Hz}, \mathrm{C}-3\right), 74.35$ (C-5), 64.85 (C-6), 52.45 (d, $\left.J_{\mathrm{C}-\mathrm{P}}=5.4 \mathrm{~Hz}, \mathrm{OMe}\right)$, $52.20\left(\mathrm{~d}, J_{\mathrm{C}-\mathrm{P}}=5.4 \mathrm{~Hz}, \mathrm{OMe}\right), 38.60\left(\mathrm{CO}-\mathrm{C}\left(\mathrm{CH}_{3}\right)_{3}, \mathrm{Piv}\right), 26.84\left(\mathrm{CO}-\mathrm{C}\left(\mathrm{CH}_{3}\right)_{3}, \mathrm{Piv}\right), 25.82$ $\left(\mathrm{C}\left(\mathrm{CH}_{3}\right)_{2}\right.$, acetonide), $25.17\left(\mathrm{C}\left(\mathrm{CH}_{3}\right)_{2}\right.$, acetonide), $21.22\left(\mathrm{CO}-\mathrm{CH}_{3}, \mathrm{Ac}\right) ;{ }^{31} \mathrm{P}-\mathrm{NMR}(101 \mathrm{MHz}$, $\left.\mathrm{CDCl}_{3}\right) \delta$ 19.14; MS $\left(\mathrm{CI}-\mathrm{NH}_{3}\right) \mathrm{m} / \mathrm{z} 451(100 \%)[\mathrm{M}+\mathrm{H}]^{+}, 468(35 \%)\left[\mathrm{M}+\mathrm{NH}_{4}\right]^{+}$; El. Anal. for $\mathrm{C}_{19} \mathrm{H}_{31} \mathrm{O}_{10} \mathrm{P}$ : calc. (\%) C 50.66 H 6.94. mes. C 50.46 H 7.13.

\section{Dimethyl (2-O-acetyl-5,6-O-(dimethyl-methylidene)-3-O-pivaloyl- $\alpha$-D-galactofuranosyl)} methanephosphonate (12a). The "general procedure for exoglycals hydrogenation" was applied. After chromatography on silica gel (AcOEt), compound 12a (82\%) was isolated as a colourless oil $\left(\tilde{\alpha} \beta\right.$. 84/16). ${ }^{1} \mathrm{H}-\mathrm{NMR}\left(400 \mathrm{MHz}, \mathrm{CDCl}_{3}\right) \delta 5.14\left(\mathrm{~d}, J_{1-2}=3.4 \mathrm{~Hz}, 1 \mathrm{H}, \mathrm{H}-2_{\alpha}\right), 5.15-5.12(\mathrm{~m}$, $\left.0.46 \mathrm{H}, \mathrm{H}-2_{\beta}, \mathrm{H}-3_{\beta}\right), 4.94\left(\mathrm{~d}, J_{3-4}=3.8 \mathrm{~Hz}, 1 \mathrm{H}, \mathrm{H}-3_{\alpha}\right), 4.48\left(\mathrm{tdd}, J_{1-2}=4.1 \mathrm{~Hz}, J_{1}{ }^{\prime} \mathrm{a}, \mathrm{b}-1=7.1 \mathrm{~Hz}, J_{1-}\right.$ $\left.\mathrm{P}=11.1 \mathrm{~Hz}, 0.23 \mathrm{H}, \mathrm{H}-1_{\beta}\right), 4.36\left(\mathrm{tdd}, J_{1-2}=3.4 \mathrm{~Hz}, J_{1}{ }^{\prime} \mathrm{a}, \mathrm{b}-1=7.1 \mathrm{~Hz}, J_{1-\mathrm{P}}=10.7 \mathrm{~Hz}, 1 \mathrm{H}, \mathrm{H}-1_{\alpha}\right), 4.31$ $\left(\mathrm{q}, J_{4-5}=J_{5-6 \mathrm{a}, \mathrm{b}}=6.7 \mathrm{~Hz}, 1 \mathrm{H}, \mathrm{H}-5_{\alpha}\right), 4.04\left(\mathrm{ABX}, J_{5-6 \mathrm{a}}=6.7 \mathrm{~Hz}, J_{6 \mathrm{a}-6 \mathrm{~b}}=8.4 \mathrm{~Hz}, 0.23 \mathrm{H}, \mathrm{H}-6 \mathrm{a}_{\beta}\right)$, $4.03\left(\mathrm{ABX}, J_{5-6 \mathrm{a}}=6.8 \mathrm{~Hz}, J_{6 \mathrm{a}-6 \mathrm{~b}}=8.6 \mathrm{~Hz}, 1 \mathrm{H}, \mathrm{H}-6 \mathrm{a}_{\alpha}\right), 3.93\left(\mathrm{~m}, 0.23 \mathrm{H}, \mathrm{H}-4_{\beta}\right), 3.84\left(\mathrm{ABX}, J_{5-6 \mathrm{~b}}=\right.$ $\left.6.2 \mathrm{~Hz}, J_{6 \mathrm{a}-6 \mathrm{~b}}=8.6 \mathrm{~Hz}, 1.23 \mathrm{H}, \mathrm{H}-6 \mathrm{~b}_{\alpha}, \mathrm{H}-6_{\beta}\right), 3.75,3.73\left(2 \mathrm{~d}, J_{\mathrm{H}-\mathrm{P}}=11.1,11.0 \mathrm{~Hz}, 6 \mathrm{H}, \mathrm{OMe}_{\alpha}\right)$, 3.76, $3.74\left(2 \mathrm{~d}, J_{\mathrm{H}-\mathrm{P}}=11.0,10.9 \mathrm{~Hz}, 1.38 \mathrm{H}, \mathrm{OMe}_{\beta}\right), 3.75\left(\mathrm{~m}, 1 \mathrm{H}, \mathrm{H}-5_{\beta}\right), 2.26-2.12\left(\mathrm{ABXX}^{\prime}, J_{1}\right.$ 'a-

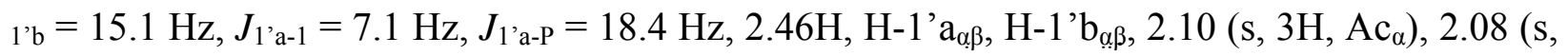

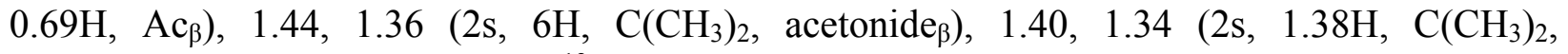
acetonide $\left.{ }_{\alpha}\right), 1.20\left(\mathrm{~s}, 11 \mathrm{H}, \mathrm{Piv}_{\alpha \beta}\right) ;{ }^{13} \mathrm{C}-\mathrm{NMR}\left(100 \mathrm{MHz}, \mathrm{CDCl}_{3}\right) \delta 177.03\left(\mathrm{C}=\mathrm{O}, \mathrm{Piv}_{\alpha \beta}\right), 169.74$

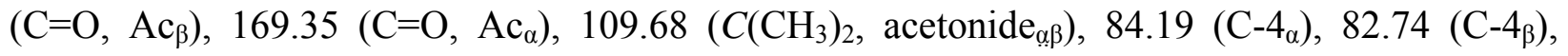
$80.69\left(\mathrm{~d}, J_{2-\mathrm{P}}=12.0 \mathrm{~Hz}, \mathrm{C}-2_{\beta}\right), 78.45\left(\mathrm{C}-3_{\beta}\right), 78.37\left(\mathrm{C}-3_{\alpha}\right), 77.70\left(\mathrm{~d}, J_{1-\mathrm{P}}=2.9 \mathrm{~Hz}, \mathrm{C}-1_{\beta}\right), 77.41$ $\left(\mathrm{d}, J_{2-\mathrm{P}}=8.1 \mathrm{~Hz}, \mathrm{C}-2_{\alpha}\right), 75.65\left(\mathrm{C}-5_{\beta}\right), 75.29\left(\mathrm{C}-5_{\alpha}\right), 75.16\left(\mathrm{C}-1_{\alpha}\right), 65.40\left(\mathrm{C}-6_{\alpha}\right), 65.37\left(\mathrm{C}-6_{\beta}\right)$, $52.73\left(\mathrm{~d}, J_{\mathrm{C}-\mathrm{P}}=6.2 \mathrm{~Hz}, \mathrm{OMe}_{\alpha}\right), 52.55\left(\mathrm{~d}, J_{\mathrm{C}-\mathrm{P}}=6.5 \mathrm{~Hz}, \mathrm{OMe}_{\beta}\right), 52.23\left(\mathrm{~d}, J_{\mathrm{C}-\mathrm{P}}=6.7 \mathrm{~Hz}, \mathrm{OMe}_{\beta}\right)$, $52.14\left(\mathrm{~d}, J_{\mathrm{C}-\mathrm{P}}=6.3 \mathrm{~Hz}, \mathrm{OMe}_{\alpha}\right), 38.48\left(\mathrm{CO}-\mathrm{C}\left(\mathrm{CH}_{3}\right)_{3}, \mathrm{Piv}_{\alpha \cdot \beta}\right), 28.67\left(\mathrm{~d}, J_{1}{ }^{\prime}-\mathrm{P}=140.7 \mathrm{~Hz}, \mathrm{C}-1^{\prime}{ }_{\beta}\right)$, $\left.26.84\left(\mathrm{CO}-\mathrm{C}\left(\mathrm{CH}_{3}\right)_{3}, \mathrm{Piv}_{\beta}\right), 26.82\left(\mathrm{CO}-\mathrm{C}\left(\mathrm{CH}_{3}\right)_{3}, \mathrm{Piv}_{\alpha}\right), 26.19,25.21\left(\mathrm{C}_{\left(\mathrm{CH}_{3}\right)_{2} \text {, acetonide }}\right)\right), 26.09$, $25.11\left(\mathrm{C}_{\left(\mathrm{CH}_{3}\right)_{2} \text {, acetonide }}\right), 24.78\left(\mathrm{~d}, J_{1}{ }^{\prime}-\mathrm{P}=141.8 \mathrm{~Hz}, \mathrm{C}-1^{\prime}{ }_{\alpha}\right), 20.70\left(\mathrm{CO}-\mathrm{CH}_{3}, \mathrm{Ac}_{\beta}\right), 20.59$ $\left(\mathrm{CO}-\mathrm{CH}_{3}, \mathrm{Ac}_{\alpha}\right) ;{ }^{31} \mathrm{P}-\mathrm{NMR}\left(101 \mathrm{MHz}, \mathrm{CDCl}_{3}\right) \delta 29.69(\alpha), 28.89(\beta) ; \mathrm{MS}\left(\mathrm{CI}_{-N H_{3}}\right) \mathrm{m} / \mathrm{z} 453[\mathrm{M}+$ 
$\mathrm{H}]^{+}$; HRMS for $\mathrm{C}_{19} \mathrm{H}_{34} \mathrm{O}_{10} \mathrm{P}$ calc. 453.18 .90 mes. 453.1894 .

\section{(1(1')Z)-2,5,6-Tri-O-tert-butyldimethylsilyl-1-(dimethoxyphosphoryl)methylidene-D-}

galactofuranose. To a solution of exoglycal $8(940 \mathrm{mg}, 1.32 \mathrm{mmol})$ in anhydrous $\mathrm{MeOH}(30$ $\mathrm{mL}$ ) was added at $0^{\circ} \mathrm{C} \mathrm{NaH}(160 \mathrm{mg}, 60 \%$ in mineral oil). The solution was stirred 12 hours at room temperature and quenched by adding silica gel $(3 \mathrm{~g})$. The suspension was filtrated, concentrated and chromatographed on silica gel (Cy/AcOEt 3/1) to yield $13(530 \mathrm{mg}, 68 \%)$ as viscous solid. $[\alpha]_{\mathrm{D}}{ }^{22}-7.3\left(\mathrm{c} 0.46, \mathrm{CHCl}_{3}\right)$; m.p. 91-92 ${ }^{\circ} \mathrm{C} ;{ }^{1} \mathrm{H}-\mathrm{NMR}\left(400 \mathrm{MHz}, \mathrm{CDCl}_{3}\right) \delta 4.65$ $\left(\mathrm{ddd}, J_{1^{\prime}-2}=1.7 \mathrm{~Hz}, J_{2-3}=5.9 \mathrm{~Hz}, J_{2-\mathrm{P}}=4.3 \mathrm{~Hz}, 1 \mathrm{H}, \mathrm{H}-2\right), 4.60\left(\mathrm{dd}, J_{1^{\prime}-2}=1.7 \mathrm{~Hz}, J_{1^{\prime}-\mathrm{P}}=10.4 \mathrm{~Hz}\right.$, 1H, H-1'), 4.04 (m, 2H, H-3, H-5), 3.83 (d, J=2.1 Hz, 1H, H-4), 3.79-3.76 (m, 3H, 1H, H-6a, H-6b, OH-2), 3.72 (2d, $\left.J_{\mathrm{H}-\mathrm{P}}=11.1 \mathrm{~Hz}, 6 \mathrm{H}, \mathrm{OMe}\right), 0.96$ (s, 9H, Si-tBu), 0.94 (s, 9H, Si-tBu), 0.92 (s, 9H, Si-tBu), 0.21 (s, 3H, Si-Me), 0.19 (s, 6H, Si-Me), 0.16 (s, 3H, Si-Me), 0.15 (s, 3H, Si-Me), 0.14 (s, 3H, Si-Me); ${ }^{13} \mathrm{C}-\mathrm{NMR}\left(100 \mathrm{MHz}, \mathrm{CDCl}_{3}\right) \delta 172.19\left(\mathrm{~d}, J_{1-\mathrm{P}}=2.1 \mathrm{~Hz}, \mathrm{C}-1\right), 86.47(\mathrm{C}-4)$, $79.59\left(\mathrm{~d}, J_{1}{ }^{\prime}-\mathrm{P}=197.1 \mathrm{~Hz}, \mathrm{C}-1^{\prime}\right), 77.46\left(\mathrm{~d}, J_{2-\mathrm{P}}=13.1 \mathrm{~Hz}, \mathrm{C}-2\right), 75.11$ (C-3), 73.06 (C-5), 64.59 $(\mathrm{C}-6), 51.80\left(\mathrm{~d}, J_{\mathrm{C}-\mathrm{P}}=5.5 \mathrm{~Hz}, \mathrm{OMe}\right), 51.78\left(\mathrm{~d}, J_{\mathrm{C}-\mathrm{P}}=5.3 \mathrm{~Hz}, \mathrm{OMe}\right), 25.54\left(\mathrm{Si}-\mathrm{C}\left(\mathrm{CH}_{3}\right)_{3}\right), 25.52$ $\left(\mathrm{Si}-\mathrm{C}\left(\mathrm{CH}_{3}\right)_{3}\right), 25.51\left(\mathrm{Si}-\mathrm{C}\left(\mathrm{CH}_{3}\right)_{3}\right), 17.89\left(\mathrm{Si}-\mathrm{C}\left(\mathrm{CH}_{3}\right)_{3}\right), 17.80\left(\mathrm{Si}-\mathrm{C}\left(\mathrm{CH}_{3}\right)_{3}\right), 17.78\left(\mathrm{Si}-\mathrm{C}\left(\mathrm{CH}_{3}\right)_{3}\right)$, 4.54 (Si-Me), -5.13 (Si-Me), -5.20 (Si-Me), -5.24 (Si-Me), -5.61 (Si-Me), -5.77 (Si-Me); ${ }^{31} \mathrm{P}-$ NMR $\left(101 \mathrm{MHz}, \mathrm{CDCl}_{3}\right) \delta$ 21.88; MS $\left(\mathrm{CI}_{-N H}\right): \mathrm{m} / \mathrm{z} 627(100 \%)[\mathrm{M}+\mathrm{H}]^{+}, 644(25 \%)$ $\left[\mathrm{M}+\mathrm{NH}_{4}\right]^{+}$; El. Anal. for $\mathrm{C}_{27} \mathrm{H}_{59} \mathrm{O}_{8} \mathrm{PSi}_{3}$ calc. (\%) C 51.72 H 9.48. mes. C $51.70 \mathrm{H} \mathrm{9.47.}$

\section{Dimethyl (2,5,6-tri-O-tert-butyldimethylsilyl-a-D-galactofuranosyl) methanephosphonate}

(13a). The "general procedure for exoglycals hydrogenation" was applied. After chromatography on silica gel (Cy/AcOEt 2/1), compound 13a (89\%) was isolated as a colourless oil.

${ }^{1} \mathrm{H}-\mathrm{NMR}\left(400 \mathrm{MHz}, \mathrm{CDCl}_{3}\right) \delta 4.26\left(\mathrm{tdd}, J_{1-2}=4.8 \mathrm{~Hz}, J_{1}{ }^{\prime} \mathrm{a}-1=4.8 \mathrm{~Hz}, J_{1^{\prime} \mathrm{b}-1}=7.8 \mathrm{~Hz}, J_{1-\mathrm{P}}=10.7\right.$ $\mathrm{Hz}, 1 \mathrm{H}, \mathrm{H}-1), 4.14\left(\mathrm{dd}, J_{1-2}=4.8 \mathrm{~Hz}, J_{2-3}=3.0 \mathrm{~Hz}, 1 \mathrm{H}, \mathrm{H}-2\right), 3.94\left(\mathrm{dd}, J_{2-3}=3.0 \mathrm{~Hz}, J_{3-4}=6.6\right.$ $\mathrm{Hz}, 1 \mathrm{H}, \mathrm{H}-3), 3.78$ (d, $\left.J_{\mathrm{H}-\mathrm{P}}=11.0 \mathrm{~Hz}, 3 \mathrm{H}, \mathrm{OMe}\right), 3.77$ (d, $\left.J_{\mathrm{H}-\mathrm{P}}=10.8 \mathrm{~Hz}, 3 \mathrm{H}, \mathrm{OMe}\right), 3.76(\mathrm{~m}, 1 \mathrm{H}$, $\mathrm{H}-5), 3.71(\mathrm{~m}, 2 \mathrm{H}, \mathrm{H}-6 \mathrm{a}, \mathrm{H}-6 \mathrm{~b}), 3.56$ (t, $\left.J_{3-4}=J_{4-5}=6.6 \mathrm{~Hz}, 1 \mathrm{H}, \mathrm{H}-4\right), 2.81\left(\mathrm{~d}, J_{\mathrm{OH}-3}=1.9 \mathrm{~Hz}\right.$, $1 \mathrm{H}, \mathrm{OH}-3), 2.21$ (ABXX', $J_{1}$ 'a-1'b $=15.8 \mathrm{~Hz}, J_{1}$ 'a-1 $=4.8 \mathrm{~Hz}, J_{1}$ 'a-P $=18.7 \mathrm{~Hz}, 1 \mathrm{H}, \mathrm{H}-1$ 'a), 2.08

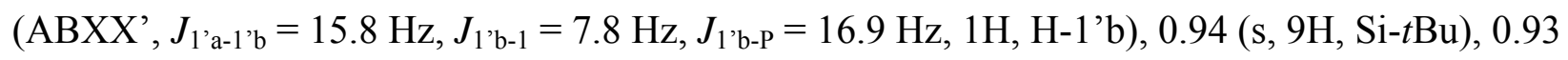
(s, 9H, Si-tBu), 0.92 (s, 9H, Si-tBu), 0.15 (s, 3H, Si-Me), 0.13 (s, 3H, Si-Me), 0.12 (s, 3H, Si$\mathrm{Me}$ ), 0.12 (s, 6H, Si-Me), 0.11 (s, 3H, Si-Me); ${ }^{13} \mathrm{C}-\mathrm{NMR}\left(100 \mathrm{MHz}, \mathrm{CDCl}_{3}\right) \delta 86.37$ (C-4), 79.93 $(\mathrm{C}-3), 79.42\left(\mathrm{~d}, J_{2-\mathrm{P}}=10.1 \mathrm{~Hz}, \mathrm{C}-2\right), 75.47\left(\mathrm{~d}, J_{1-\mathrm{P}}=2.7 \mathrm{~Hz}, \mathrm{C}-1\right), 73.78(\mathrm{C}-5), 65.93(\mathrm{C}-6)$, $52.37\left(\mathrm{~d}, J_{\mathrm{C}-\mathrm{P}}=6.4 \mathrm{~Hz}, \mathrm{OMe}\right), 52.26\left(\mathrm{~d}, J_{\mathrm{C}-\mathrm{P}}=6.5 \mathrm{~Hz}, \mathrm{OMe}\right), 25.94\left(2 \mathrm{Si}-\mathrm{C}\left(\mathrm{CH}_{3}\right)_{3}\right), 25.86(\mathrm{Si}-$ $\left.\mathrm{C}\left(\mathrm{CH}_{3}\right)_{3}\right), 25.72\left(\mathrm{~d}, J_{1}{ }^{\prime}-\mathrm{P}=143.4 \mathrm{~Hz}, \mathrm{C}-1^{\prime}\right), 18.32\left(\mathrm{Si}-\mathrm{C}\left(\mathrm{CH}_{3}\right)_{3}\right), 18.26\left(\mathrm{Si}-\mathrm{C}\left(\mathrm{CH}_{3}\right)_{3}\right), 18.10(\mathrm{Si}-$ $\left.\mathrm{C}\left(\mathrm{CH}_{3}\right)_{3}\right),-4.44$ (2Si-Me), -4.57 (2Si-Me), -4.73 (2Si-Me); ${ }^{31} \mathrm{P}-\mathrm{NMR}\left(101 \mathrm{MHz}, \mathrm{CDCl}_{3}\right) \delta 32.61$; MS $\left(\mathrm{CI}-\mathrm{NH}_{3}\right) \mathrm{m} / \mathrm{z} 629(85 \%)[\mathrm{M}+\mathrm{H}]^{+}, 646(100 \%)\left[\mathrm{M}+\mathrm{NH}_{4}\right]^{+}$; El. Anal. for $\mathrm{C}_{27} \mathrm{H}_{61} \mathrm{O}_{8} \mathrm{PSi}_{4}$ calc. (\%) C 51.56 H 9.77. mes. C 51.76 H 9.56.

\section{(1(1')Z)-2,3,6-Tri-O-tert-butyldimethylsilyl-1-(dimethoxyphosphoryl)methylidene-D-}

galactofuranose (14). The general procedure for preparing exoglycals was followed starting from known lactone. ${ }^{10} \mathrm{~A}$ white solid was obtained $(63 \%)$. $[\alpha]_{\mathrm{D}}{ }^{24}+50.3\left(\mathrm{c} 1.0, \mathrm{CHCl}_{3}\right)$; m.p. 95$96^{\circ} \mathrm{C} ;{ }^{1} \mathrm{H}-\mathrm{NMR}\left(400 \mathrm{MHz}, \mathrm{CDCl}_{3}\right) \delta 4.68\left(\mathrm{dd}, J_{1^{\prime}-2}=1.5 \mathrm{~Hz}, J_{1}{ }^{\prime}-\mathrm{P}=12.0 \mathrm{~Hz}, 1 \mathrm{H}, \mathrm{H}-1^{\prime}\right), 4.55$ $\left(\mathrm{ddd}, J_{1^{\prime}-2}=1.5 \mathrm{~Hz}, J_{2-\mathrm{P}}=4.0 \mathrm{~Hz}, J_{2-3}=6.3 \mathrm{~Hz}, 1 \mathrm{H}, \mathrm{H}-2\right), 4.45\left(\mathrm{~d}, J_{3-4}=5.9 \mathrm{~Hz}, 1 \mathrm{H}, \mathrm{H}-4\right), 4.35$ 
$\left(\mathrm{t}, J_{2-3}=J_{3-4}=6.3 \mathrm{~Hz}, 1 \mathrm{H}, \mathrm{H}-3\right), 3.76\left(\mathrm{~d}, J_{\mathrm{H}-\mathrm{P}}=11.6 \mathrm{~Hz}, 3 \mathrm{H}, \mathrm{OCH}_{3}\right), 3.74\left(\mathrm{~d}, J_{\mathrm{H}-\mathrm{P}}=11.3 \mathrm{~Hz}, 3 \mathrm{H}\right.$, $\mathrm{OCH}_{3}$ ), 3.81-3.69 (m, 3H, H-5, H-6a, H-6b), 0.98 (s, 9H, Si-tBu), 0.93 (s, 9H, Si-tBu), 0.92 (s, 9H, Si-tBu), 0.18 (s, 3H, Si-Me), 0.17 (s, 3H, Si-Me), 0.14 (s, 3H, Si-Me), 0.09 (s, 9H, 3Si-Me); ${ }^{13} \mathrm{C}-\mathrm{NMR}\left(100 \mathrm{MHz}, \mathrm{CDCl}_{3}\right) \delta 174.31\left(\mathrm{~d}, J_{1-\mathrm{P}}=2.0 \mathrm{~Hz}, \mathrm{C}-1\right), 84.71(\mathrm{C}-4), 82.82\left(\mathrm{~d}, J_{1}{ }^{\prime}-\mathrm{P}=193.3\right.$ Hz, C-1'), 78.46 (d, $\left.J_{2-\mathrm{P}}=12.5 \mathrm{~Hz}, \mathrm{C}-2\right), 75.80$ (C-3), 70.43 (C-5), 62.91 (C-6), 52.44 (d, $J_{\mathrm{C}-\mathrm{P}}=$ $\left.5.6 \mathrm{~Hz}, \mathrm{OCH}_{3}\right), 51.99\left(\mathrm{~d}, J_{\mathrm{C}-\mathrm{P}}=5.5 \mathrm{~Hz}, \mathrm{OCH}_{3}\right), 25.82\left(\mathrm{Si}-\mathrm{C}\left(\mathrm{CH}_{3}\right)_{3}\right), 25.77\left(\mathrm{Si}-\mathrm{C}\left(\mathrm{CH}_{3}\right)_{3}\right), 25.69$ $\left(\mathrm{Si}-\mathrm{C}\left(\mathrm{CH}_{3}\right)_{3}\right), 18.16\left(\mathrm{Si}-\mathrm{C}\left(\mathrm{CH}_{3}\right)_{3}\right), 17.86\left(\mathrm{Si}-\mathrm{C}\left(\mathrm{CH}_{3}\right)_{3}\right), 17.82\left(\mathrm{Si}-\mathrm{C}\left(\mathrm{CH}_{3}\right)_{3}\right),-4.05(\mathrm{Si}-\mathrm{Me}),-4.19$ (Si-Me), -4.29 (Si-Me), -4.58 (Si-Me), -5.44 (Si-Me), -5.57 ( $\mathrm{Si}-\mathrm{Me})$; ${ }^{31} \mathrm{P}-\mathrm{NMR}$ (101 MHz, $\left.\mathrm{CDCl}_{3}\right) \delta$ 22.60; MS (IC-NH 3$) \mathrm{m} / \mathrm{z} 627(100 \%)[\mathrm{M}+\mathrm{H}]^{+}$, $644(70 \%)\left[\mathrm{M}+\mathrm{NH}_{4}\right]^{+}$; El. Anal. for $\mathrm{C}_{27} \mathrm{H}_{59} \mathrm{O}_{8} \mathrm{PSi}_{3}$ calc. (\%) C $51.72 \mathrm{H}$ 9.48. mes. C $51.57 \mathrm{H} 9.64$.

Dimethyl (2,3,6-tri-O-tert-butyldimethylsilyl- $\alpha$-D-galactofuranosyl) methanephosphonate (14a). The "general procedure for exoglycals hydrogenation" was applied. After chromatography on silica gel (Cy/AcOEt 3/1), compounds 14a (76\%) and 14b (10\%) were isolated as colourless oils. $[\alpha]_{\mathrm{D}}{ }^{19}-2.5\left(\mathrm{c} 0.4, \mathrm{CHCl}_{3}\right) ;{ }^{1} \mathrm{H}-\mathrm{NMR}\left(400 \mathrm{MHz}, \mathrm{CDCl}_{3}\right) \delta 4.38\left(\right.$ tdd, $J_{1-2}=3.7 \mathrm{~Hz}, J_{1}$ 'a-1 $=$ $\left.8.0 \mathrm{~Hz}, J_{1^{\prime} \mathrm{b}-1}=4.4 \mathrm{~Hz}, J_{1-\mathrm{P}}=12.3 \mathrm{~Hz}, 1 \mathrm{H}, \mathrm{H}-1\right), 4.16\left(\mathrm{dd}, J_{2-3}=3.2 \mathrm{~Hz}, J_{3-4}=2.5 \mathrm{~Hz}, 1 \mathrm{H}, \mathrm{H}-3\right)$, $4.03\left(\mathrm{dd}, J_{3-4}=2.5 \mathrm{~Hz}, J_{4-5}=1.8 \mathrm{~Hz}, 1 \mathrm{H}, \mathrm{H}-4\right), 3.96\left(\mathrm{dd}, J_{2-3}=3.2 \mathrm{~Hz}, 1 \mathrm{H}, \mathrm{H}-2\right), 3.79\left(2 \mathrm{~d}, J_{\mathrm{H}-\mathrm{P}}=\right.$ $10.9 \mathrm{~Hz}, 6 \mathrm{H}, \mathrm{OMe}), 3.70-3.59$ (m, 3H, H-5, H-6a, H-6b), 2.19 (ABXX', $J_{1}$ 'a-1'b $=15.4 \mathrm{~Hz}, J_{1}$ 'a-1 $=$ $8.0 \mathrm{~Hz}, J_{1^{\prime} \mathrm{a}-\mathrm{P}}=16.9 \mathrm{~Hz}, 1 \mathrm{H}, \mathrm{H}-1$ 'a), $2.00\left(\mathrm{ABXX}, J_{1^{\prime} \mathrm{a}-1{ }^{\prime} \mathrm{b}}=15.4 \mathrm{~Hz}, J_{1^{\prime} \mathrm{b}-1}=4.4 \mathrm{~Hz}, J_{1^{\prime} \mathrm{b}-\mathrm{P}}=19.7\right.$ Hz, 1H, H-1'b), 0.94 (s, 9H, Si-tBu), 0.91 (s, 9H, Si-tBu), 0.9 (s, 9H, Si-tBu), 0.17 (s, 3H, Si$\mathrm{Me}$ ), 0.14 (s, 3H, Si-Me), 0.12 (s, 3H, Si-Me), 0.11 (s, 3H, Si-Me), 0.07 (s, 6H, Si-Me); ${ }^{13} \mathrm{C}-$ NMR (100 MHz, CDCl $) \delta 84.99(\mathrm{C}-4), 79.50(\mathrm{C}-3), 79.47$ (d, $\left.J_{2-\mathrm{P}}=10.9 \mathrm{~Hz}, \mathrm{C}-2\right), 75.33$ (d, $J_{1-\mathrm{P}}$ $=3.5 \mathrm{~Hz}, \mathrm{C}-1), 71.08(\mathrm{C}-5), 63.64(\mathrm{C}-6), 52.53\left(\mathrm{~d}, J_{\mathrm{C}-\mathrm{P}}=6.3 \mathrm{~Hz}, \mathrm{OMe}\right), 52.35\left(\mathrm{~d}, J_{\mathrm{C}-\mathrm{P}}=6.2 \mathrm{~Hz}\right.$, $\mathrm{OMe}), 25.87\left(\mathrm{Si}-\mathrm{C}\left(\mathrm{CH}_{3}\right)_{3}\right), 25.80\left(\mathrm{~d}, J_{1^{\prime}-\mathrm{P}}=142.4 \mathrm{~Hz}, \mathrm{C}-1^{\prime}\right), 25.78\left(\mathrm{Si}-\mathrm{C}\left(\mathrm{CH}_{3}\right)_{3}\right), 25.69(\mathrm{Si}-$ $\left.\mathrm{C}\left(\mathrm{CH}_{3}\right)_{3}\right), 18.23\left(\mathrm{Si}-\mathrm{C}\left(\mathrm{CH}_{3}\right)_{3}\right), 18.01\left(\mathrm{Si}-\mathrm{C}\left(\mathrm{CH}_{3}\right)_{3}\right), 17.81\left(\mathrm{Si}-\mathrm{C}\left(\mathrm{CH}_{3}\right)_{3}\right),-4.41(\mathrm{Si}-\mathrm{Me}),-4.46(\mathrm{Si}-$ $\mathrm{Me}$ ), -4.55 (Si-Me), -4.91 (Si-Me), -5.39 (Si-Me), -5.48 (Si-Me); ${ }^{31} \mathrm{P}-\mathrm{NMR}\left(101 \mathrm{MHz}, \mathrm{CDCl}_{3}\right) \delta$ 32.50; MS (DIC-NH $\left.)_{3}\right) \mathrm{m} / \mathrm{z} 629(100 \%)[\mathrm{M}+\mathrm{H}]^{+}, 646(60 \%)\left[\mathrm{M}+\mathrm{NH}_{4}\right]^{+}$; El. Anal. for $\mathrm{C}_{27} \mathrm{H}_{61} \mathrm{O}_{8} \mathrm{PSi}_{3}$ calc. (\%) C $51.56 \mathrm{H}$ 9.77. mes. C $51.59 \mathrm{H} \mathrm{9.70.}$

Dimethyl (2,3,6-tri-O-tert-butyldimethylsilyl-1-enyl-D-galactofuranosyl)methanephosphonate (14b). ${ }^{1} \mathrm{H}-\mathrm{NMR}\left(400 \mathrm{MHz}, \mathrm{CDCl}_{3}\right) \delta 4.83\left(\mathrm{dd}, J_{3-4}=2.0 \mathrm{~Hz}, J_{4-5}=6.5 \mathrm{~Hz}, 1 \mathrm{H}, \mathrm{H}-4\right)$, $4.42\left(\mathrm{~d}, J_{3-4}=2.0 \mathrm{~Hz}, 1 \mathrm{H}, \mathrm{H}-3\right), 3.82\left(\mathrm{~d}, J_{\mathrm{H}-\mathrm{P}}=11.2 \mathrm{~Hz}, 3 \mathrm{H}, \mathrm{OMe}\right), 3.78\left(\mathrm{~d}, J_{\mathrm{H}-\mathrm{P}}=11.0 \mathrm{~Hz}, 3 \mathrm{H}\right.$, OMe), 3.78-3.67 (m, 3H, H-5, H-6a, H-6b), 3.01 (ABX, $J_{1 ' a-1 ’ b}=15.7 \mathrm{~Hz}, J_{1}$ 'a-P $=19.0 \mathrm{~Hz}, 1 \mathrm{H}$, H-1'a), 2.67 (ABX, $J_{1^{\prime} \mathrm{a}-1 ' \mathrm{~b}}=15.7 \mathrm{~Hz}, J_{1}$ 'b-P $=21.2 \mathrm{~Hz}, 1 \mathrm{H}, \mathrm{H}-1$ 'b), 0.98 (s, 9H, Si-tBu), 0.93 (s, 9H, Si-tBu), 0.92 (s, 9H, Si-tBu), 0.22 (s, 3H, Si-Me), 0.20 (s, 3H, Si-Me), 0.13 (2s, 6H, 2Si-Me), 0.08 (s, 6H, 2Si-Me); ${ }^{13} \mathrm{C}-\mathrm{NMR}\left(100 \mathrm{MHz}, \mathrm{CDCl}_{3}\right) \delta 134.61$ (d, $J_{1,2-\mathrm{P}}=14.6 \mathrm{~Hz}, \mathrm{C}-1$ and C-2), 134.38 (d, $J_{1,2-\mathrm{P}}=12.4 \mathrm{~Hz}, \mathrm{C}-1$ and C-2), $84.56(\mathrm{C}-4), 77.80$ (d, $\left.J_{3-\mathrm{P}}=2.8 \mathrm{~Hz}, \mathrm{C}-3\right), 74.09$ (C-5), $63.09(\mathrm{C}-6), 52.73\left(\mathrm{~d}, J_{\mathrm{C}-\mathrm{P}}=6.6 \mathrm{~Hz}, \mathrm{OMe}\right), 52.71\left(\mathrm{~d}, J_{\mathrm{C}-\mathrm{P}}=6.8 \mathrm{~Hz}, \mathrm{OMe}\right), 25.92\left(\mathrm{Si}-\mathrm{C}\left(\mathrm{CH}_{3}\right)_{3}\right)$, $25.87\left(\mathrm{Si}-\mathrm{C}\left(\mathrm{CH}_{3}\right)_{3}\right), 25.63\left(\mathrm{Si}-\mathrm{C}\left(\mathrm{CH}_{3}\right)_{3}\right), 23.31\left(\mathrm{~d}, J_{1}{ }^{\prime}-\mathrm{P}=139.8 \mathrm{~Hz}, \mathrm{C}-1^{\prime}\right), 18.23\left(\mathrm{Si}-\mathrm{C}\left(\mathrm{CH}_{3}\right)_{3}\right)$, $17.99\left(\mathrm{Si}-\mathrm{C}\left(\mathrm{CH}_{3}\right)_{3}\right), 17.88\left(\mathrm{Si}-\mathrm{C}\left(\mathrm{CH}_{3}\right)_{3}\right),-3.66(\mathrm{Si}-\mathrm{Me}),-4.08(\mathrm{Si}-\mathrm{Me}),-4.18(\mathrm{Si}-\mathrm{Me}),-4.26(\mathrm{Si}-$ $\mathrm{Me}$ ), -5.42 (Si-Me), -5.54 (Si-Me); ${ }^{31} \mathrm{P}-\mathrm{NMR}\left(101 \mathrm{MHz}, \mathrm{CDCl}_{3}\right) \delta$ 29.44; MS (FAB+) m/z 626 
(100\%) [M], $649(90 \%)[\mathrm{M}+\mathrm{Na}]^{+}$; HRMS for $\mathrm{C}_{27} \mathrm{H}_{59} \mathrm{O}_{8} \mathrm{PSi}_{3}$ calc. 626.3255 mes. 626.3239; HRMS for $\mathrm{C}_{27} \mathrm{H}_{59} \mathrm{O}_{8} \mathrm{PSi}_{3} \mathrm{Na}$ calc. 649.3153 mes. 649.3151 .

\section{Acknowledgements}

We warmly thank Professor Krief and his team for sharing their facilities and for their stimulating scientific discussions. This work was supported by the Centre National de la Recherche Scientifique (CNRS, France), by the Ministere Delegue à la Recherche et aux Nouvelles Technologies (PhD grant to A.C.) as well as the University of Namur (post-doctoral grants to W.P.).

\section{References and Notes}

1. Caravano, A.; Mengin-Lecreulx, D.; Brondello, J.-M.; Vincent, S. P.; Sinaÿ, P. Chem. Eur. J. 2003, 9, 5888.

2. Caravano, A.; Sinaÿ, P.; Vincent, S. P. Bioorg. Med. Chem. Lett. 2006, 16, 1123.

3. Caravano, A.; Dohi, H.; Sinaÿ, P.; Vincent, S. P. Chem. Eur. J. 2006, 11, 3114.

4. Caravano, A.; Vincent, S. P.; Sinä̈, P. Chem. Commun. 2004, 1216.

5. Burkart, M. D.; Vincent, S. P.; Düffels, A.; Murray, B. W.; Ley, S. V.; Wong, C.-H. Bioorg. Med. Chem. 2000, 8, 1937.

6. Stork, G.; Kahne, D. J. Am. Chem. Soc. 1983, 105, 1072.

7. Brown, J. M. Angew. Chem., Int. Ed. 1987, 26, 190.

8. Smith, M. K. E.; Derrien, N.; Lloyd, M. C.; Taylor, S. J. C.; Chaplin, D. A.; Mccague, R. Tetrahedron Lett. 2001, 42, 1347.

9. Paquette, L. A.; Peng, X.; Bondar, D. Org. Lett. 2002, 4, 937.

10. Krülle, T.; Fuente, C. D. L.; Pickering, L.; Aplin, R. T.; Tsitsanou, K. E.; Zographos, S. E.; Oikonomakos, N. G.; Nash, R. J.; Griffiths, R. C.; Fleet, G. W. J. Tetrahedron: Asymmetry 1997, 8, 3807.

11. Yang, W.-B.; Wu, C.-Y.; Chang, C.-C.; Wang, S.-H.; Teo, C.-F.; Lin, C.-H. Tetrahedron Lett. 2001, 42, 6907.

12. Yang, W.-B.; Yang, Y.-Y.; Gu, Y.-F.; Wang, S.-H.; Chang, C.-F.; Lin, C.-H. J. Org. Chem. 2002, 67, 3773.

13. Bundle, D. R.; Lemieux, R. U. Methods Carbohydr. Chem. 1976, 7, 79.

14. Varela, O.; Marino, M.; Lederkremer, R. M. D. Carbohydr. Res. 1986, 155, 247. 